\title{
Longitudinal Analyses Reveal That Aging-related Alterations in the Intestinal Environment Lead to Gut Dysbiosis With the Potential to Induce Obesity
}

\section{Yumiko Nakanishi}

RIKEN Center for Integrative Medical Sciences

\section{Ryouko Nozu}

Central Institute for Experimental Animals

\section{Masami Ueno}

Central Institute for Experimental Animals

\section{Kyoji Hioki}

Central Institute for Experimental Animals

\section{Chiharu Ishii}

Keio University: Keio Gijuku Daigaku

Shinnosuke Murakami

Keio University: Keio Gijuku Daigaku

\section{Kenta Suzuki}

Riken BioResource Research Center

\section{Yuyo Ka}

Central Institute for Experimental Animals

\section{Tomoyuki Ogura}

Central Institute for Experimental Animals

Ayumi Ito

Riken Center for Integrative Medical Sciences

Naoko Tachibana

RIKEN Center for Integrative Medical Sciences

\section{Tamotsu Kato}

Riken Center for Integrative Medical Sciences

Akiyoshi Hirayama

Keio University: Keio Gijuku Daigaku

\section{Masahiro Sugimoto}

Keio University: Keio Gijuku Daigaku

\section{Tomoyoshi Soga}

Keio University: Keio Gijuku Daigaku

Mamoru Ito 
Central Institute for Experimental Animals

Masaru Tomita

Keio University: Keio Gijuku Daigaku

\section{Hiroshi Ohno}

Riken Center for Integrative Medical Sciences

Shinji Fukuda ( $\nabla$ sfukuda@sfc.keio.ac.jp)

RIKEN Center for Integrative Medical Sciences https://orcid.org/0000-0001-5161-9880

\section{Research}

Keywords: Aging, Gut microbiota, Metabolites, Longitudinal analyses, dysbiosis

Posted Date: December 7th, 2020

DOl: https://doi.org/10.21203/rs.3.rs-119480/v1

License: (c) (i) This work is licensed under a Creative Commons Attribution 4.0 International License. Read Full License 


\section{Abstract}

Background: Aging is a progressive decline of cellular functions that ultimately affects whole-body homeostasis. Alterations in the gut microbiota associated with aging have been reported, however the molecular basis of the relationships between host aging and the gut microbiota is poorly understood.

Result: By using longitudinal microbiome and metabolome characterization, we show that the agingrelated alterations in the intestinal environment lead to gut dysbiosis with a potential to induce obesity in mice. In middle-age mice, we observed more than a 2-fold increase in fecal carbohydrates derived from dietary polysaccharides and a significant reduction of gut microbial diversity resembling the microbiota characteristic of obese mice. Consistently, fecal microbiota transplantation from middle-age specific pathogen-free (SPF) mice into young germ-free (GF) mice resulted in increased weight gain and impaired glucose tolerance.

Conclusion: Our findings provide new insights into the relationships between host aging and gut dysbiosis and may contribute to the development of a possible solution to aging-related obesity.

\section{Introduction}

Aging is characterized by the progressive functional decline of cellular functions, hence of multiple organs and tissues, which results in reduced resistance to stress, increased vulnerability to diseases and eventually culminates in death[1,2]. The aging process also seriously affects the human gut microbiota. It is accompanied by a reduction in gastrointestinal functions including intestinal motility, digestive and absorptive functions, as well as immune responses[3-9], in addition to changes in diet and lifestyle in the elderly[10,11]. Moreover, many recent studies have shown that compositional and functional abnormalities of the human gut microbiota (dysbiosis) are correlated with inflammatory disorders and metabolic syndrome, including inflammatory bowel disease[6], irritable bowel syndrome[12], type 2 diabetes (T2D) $[13,14]$ and obesity[15-17]. Notably, human studies have revealed that T2D and obesity increase in the elderly, implicating a possible contribution of the alterations in gut microbiota to the onset of age-associated diseases and their progression.

The composition of the gut microbiota is subject to a rapid change during early childhood, from breastfeeding to the weaning period. This becomes relatively stable at the species level during adult life, although this relative stability tends to be lost with aging[3,11, 18-20]. Virtually all human studies include probable confounding factors, i.e. different genetic backgrounds in combination with changes in diet, medications and lifestyle. By contrast, animal studies, especially of mice, allow more controlled experiments and analyses with pure genetic backgrounds and uniform diet and breeding environments[21-23]. The average lifespan of mice is $24-30$ months and their physiological state is defined by a frailty index[24]. Previous reports have defined age groups as 'young' (less than 6 months), 'middle' (around 20 months) and 'old' (more than 30 months), which correlate with the frailty index and body weight change[21, 24]. Body weight and fat mass increase until middle-age, despite a slight 
reduction of food intake, which is mostly due to the decrease in physical activity and muscle mass $[21,25$, 26]. At older ages, body weight gradually decreases, probably because of appetite loss and reduced food digestion and absorption, resulting in diminished energy intake with eventual weight loss[27].

Murine gut microbial composition is correlated with aging[20,23, 24]; recent reports show that older mice exhibit microbial dysbiosis, concomitantly with increased intestinal permeability (leaky gut), systemic inflammation, glucose intolerance and insulin resistance compared to younger mice [7, 28-30]. Fecal microbiota transplantation from old donor mice to young germ-free mice promotes leaky gut-associated low-grade systemic inflammation[7], indicating that ageing-associated microbiota can directly influence host physiology. Other reports showed that probiotic treatment could prevent aging-related leaky gut and inflammation in old mice [30] and that fecal microbiota transplantation from wild-type mice improved the health and lifespan in progeroid murine models[31], suggesting that intervention of the gut microbiota in old mice may improve host health and lifespan.

However, these studies were mainly based on the simple comparison of young and old subjects, instead of observing the same individuals repeatedly over time. In this study, we longitudinally analyzed fecal microbial community composition, together with fecal and plasma metabolomes, in C57BL/6J mice over a period of 72 weeks to explore the consequences of gut microbiota changes associated with aging and their influences on the host. To eliminate possible perturbation of microbial composition by external bacterial contamination, we took advantage of a radiation-sterilized diet and vinyl isolators to maintain GF and SPF mice. We found aging-associated correlations among microbial composition and the fecal and plasma metabolomes in middle-age mice. Fecal microbiota from the middle-age mice conferred an obese phenotype upon association with "young" GF mice, suggesting that the aging-associated gut microbial changes are not merely the consequence of host aging but rather could dictate aging-related phenotypes. These approaches thus allow us to achieve a comprehensive understanding of the relationships between host aging and the gut microbiota and may provide insights into the development of a solution to aging-related obesity through manipulation of the intestinal microbiota.

\section{Results}

\section{Aging in SPF mice is associated with an overall alteration in the structure of the gut microbiota}

To characterize aging-associated gut microbiota and metabolic phenotype changes, fecal and plasma samples are repetitively collected from GF and SPF mice at 3, 4, 6, 8, 12 weeks after birth, and every 4 weeks thereafter. We divided the experimental time course into 4 categories: 3 to 12 weeks (wean-young), 16 to 32 weeks (young), 36 to 52 weeks (early-middle) and 56 to 72 weeks (middle), respectively (Supplementary Fig. 1), based on the age-related murine frailty index[24] and a report showing that the murine gut microbiota changes significantly during weaning[20]. The increase of body weight in SPF mice during aging (Supplementary Fig. 2) is mostly due to an increase in fat mass, even though food intake is slightly decreased[21, 25, 26]. Weight gain in middle-age GF mice compared to middle-age SPF 
mice is probably due to the gradual cecum enlargement characteristic of GF mice because of the accumulation of hydrated dietary fiber components[32].

To assess the overall structures of the gut microbiota, we amplicon-sequenced the V1-V2 region of the 16S rRNA gene. Using $97 \%$ as the identity threshold from 118 samples with 10,000 reads per sample, 1,377 operational taxonomic units (OTUs) were identified. Principal coordinate analysis (PCoA) of weighted and unweighted UniFrac distances on fecal microbiome data revealed an alteration of the gut microbial structure of individual SPF mice with aging (Fig. 1a and b). Mean relative abundance values of multiple bacterial OTUs exhibited shifts with aging in SPF mice (Fig. 1c and Supplementary Fig. 3). Notably, the gut microbiota in wean-young mice drastically changed between 3 and 8 weeks. Hierarchical clustering analysis (HCA) of the $16 \mathrm{~S}$ rRNA fecal microbiome datasets revealed that fecal microbes were mainly separated into two clusters consisting of those decreased (Cluster 01) and increased (Cluster 02) with aging (Supplementary Fig. 4). Alpha diversity (Shannon index) in early-middle and middle-age mice declined slightly but significantly compared to wean-young mice (Fig. 1d and Supplementary Fig. 5). The microbial genera, Allobaculum, Turicibacter (now assigned as Erysipelotrichaceae family) and unclassified genus of the S24-7 family (Bacteroidales group) were significantly increased, whereas unclassified genera of the Lachnospiraceae and Ruminococcaceae family were significantly decreased with aging (Fig. 1e and Supplementary Fig. 6).

\section{Aging-associated alterations in the fecal metabolome profiles}

We next performed metabolome analysis by capillary electrophoresis-time-of-flight mass spectrometry (CE-TOFMS) and liquid chromatography-tandem mass spectrometry (LC-MS/MS) to identify ageassociated fecal metabolite changes in GF and SPF mice. This analysis identified a total of 204 metabolites in all murine feces, of which 183 and 195 metabolites were identified in GF and SPF mice, respectively. Principal component analysis (PCA) on the fecal metabolome data showed that the profiles were clustered into GF and SPF mice groups in Principal component 1 (PC1) (Fig 2a). PCA of GF and SPF mice revealed age-related change in fecal metabolites in both groups (Fig $2 b$ and $c$ ). To categorize the metabolite patterns in aging, we performed $k$-means clustering using the $z$-score values calculated by concentrations of metabolites in each type of mice (Fig 2d). Fecal metabolites of both SPF and GF mice separated by the $k$-means cluster showed change with aging; however, most of metabolites had different patterns with aging between SPF and GF mice. Comparison of the concentration of fecal metabolites from GF and SPF mice (Fig. 2d, the rightmost yellow- and green- colored heatmap) showed the large difference in the concentration of various metabolites between GF and SPF mice. Glucosamine, argininosuccinate, glyoxylate and mucate were found only in GF (Fig 2e). In contrast, 20 metabolites including butyrate were found only in SPF and gradually changed with aging (Fig 2f). The concentration of short-chain fatty acids (SCFAs: propionate, butyrate and hexanoate), vitamin B6, aspartate, alanine, lysine, cholate and sugars (maltose and maltotriose) was higher in SPF mice than in GF mice, whereas the other 14 amino acids, derivatives of amino acids and monosaccharides were lower in SPF mice (Supplementary Data 1). These results were mostly consistent with previous reports comparing fecal metabolites in GF and SPF mice[2, 33, 34]. 
The various fecal metabolites derived from SPF and GF mice changed gradually in association with aging (Fig 2d). Cluster 1, which contains 12 amino acids and their related metabolites involved in amino acid metabolism accumulated at 3 to 4 weeks and then decreased toward young age in SPF mice, whereas those in GF mice were comparable throughout the observed period. Cluster 2 included sugars and amino acids that accumulated at 4 to 12 weeks followed by a gradual reduction with aging in SPF and GF mice. Cluster 3 included nucleotides that accumulated at 4 to 12 weeks in SPF mice, whereas those in GF mice were tended to increase with aging. Clusters 4 and 5 gradually increased with aging in SPF mice. In contrast, cluster 4 was comparable with aging and cluster 5 was slightly accumulated in 3 to 12 weeks in GF mice. Cluster 4 contained amino acids and derivatives of amino acids and vitamins, whereas Cluster 5 included sugars and citrate, fumarate, malate, lactate, belonging to the citric acid cycle, that were especially increased in middle-aged SPF mice. In summary, profiles in SPF mice were clearly changed with aging compared to those in GF mice, and the pattern of alteration in fecal metabolites with aging was different between SPF mice and GF mice, implying that alterations in the intestinal environment associated with aging are likely caused by changes in the gut microbiota with age.

In the gut microbial metabolism, the most prominent microbial activity is the fermentation of dietary or host-derived components, in particular metabolism of non-digestible carbohydrates and host glycans into SCFAs and organic acids[35, 36]. Butyrate and hexanoate were significantly decreased (4- and 2-fold, respectively) in middle-aged SPF mice compared to young ones, whereas lactate was significantly increased by 2-fold in middle-age SPF mice compared to wean-young and young mice (Fig 2g). Moreover, sugars such as sucrose, maltose and maltotriose, thought to be derived from the diet, were also significantly increased more than 2-fold in middle-age SPF mice compared to younger mice (Fig $2 \mathrm{~h}$ ). Notably, maltose and maltotriose were detected mainly in SPF, but not GF mice, suggesting that these sugars might be derived from microbial digestion of dietary starch. We also found the concentration of soluble starch in feces was significantly increased in middle-age GF mice as compared to young GF mice, but was comparable across all ages in SPF mice (Supplementary Fig. 7). Considering the fecal metabolome profiles of GF mice, these notable fecal metabolome alterations in SPF mice imply that the aging-related phenotype of the intestinal environment is largely associated with modulation of the gut microbiota together with host aging.

\section{Aging-associated alterations of plasma metabolome profiles}

We further analyzed the plasma metabolome of these mice to capture age-associated plasma metabolic dynamics. This analysis identified 144 metabolites in total across all the murine plasma samples out of which 134 and 142 metabolites are identified in GF and SPF mice, respectively. PCA showed that the plasma metabolome profiles were roughly clustered into 2 groups of GF and SPF in the PC1 direction and aging-associated changes of GF and SPF plasma metabolome profiles were observed in the PC2 direction (Fig 3a). Furthermore, PCA calculated in each type of mouse showed aging-associated changes in plasma metabolome profiles in both GF and SPF mice (Fig 3b and c). K-means clustering of the plasma metabolites showed similar aging-associated shifts from weaning to middle-age between SPF and GF mice (Fig 3d). Argininosuccinate and 5-methoxy-3-indoleaceate were found in GF only (Fig 3e). In 
contrast, 10 metabolites including 5-aminovalerate and ectoine, which are thought to be mainly derived from gut microbiota[37, 38], were found only in SPF mice and most of them were gradually accumulated with aging (Fig 3f).

Amino acids and their related metabolites categorized into clusters 1, 4 and 5 were accumulated in both GF and SPF middle-aged mice (Fig 3d). The average total amino acid concentration in middle-age mice was significantly higher than that in both GF and SPF young mice (Fig $3 \mathrm{~g}$ ). Although most of the agingassociated amino acid profiles were similar between GF and SPF mice, the concentrations of glycine, serine and threonine were significantly lower in SPF mice than in GF mice almost throughout life (Supplementary Fig 8), whereas glutamine was higher in SPF mice across all ages (Fig 3h). The concentrations of pyruvate, an intermediate metabolite of glycolysis, were also significantly higher in SPF mice than in GF mice across all ages. Notably, however, the glutamate levels were comparable from young to early-middle between GF and SPF mice, and only higher in the middle-age SPF mice (Fig $3 \mathrm{~h}$ ). Cluster 2 included various types of metabolites decreasing with aging in both GF and SPF mice. Metabolites of cluster 3 were dispersed, with no change with aging.

\section{Correlation between metabolites and microbes and transition time point analyses of SPF mice with aging}

To examine aging-associated correlations among the fecal microbes, fecal metabolites and plasma metabolites in SPF mice, we calculated Spearman's rank correlation coefficients among fecal microbefecal metabolite and fecal microbe-plasma metabolite pairs in a total of 119 samples (Supplementary Fig 9 and 10). Turicibacter, Allobaculum, Bifidobacterium, unclassified S24-7 and Sutterella, categorized as cluster 02, increased with aging (Supplementary Fig. 4), whereas unclassified Lachnospiraceae, Oscillospira, unclassified Clostridiales, Ruminococcus and unclassified Ruminococcaceae, categorized cluster 01 , decreased and were highly and significantly correlated to fecal metabolites. In the correlations of fecal microbes and plasma metabolites, Turicibacterwas highly and significantly correlated. Moreover, Procrustes analysis between the fecal metabolome and the fecal microbiome profiles of weighted UniFrac or unweighted UniFrac showed that the aging profiles in fecal metabolome and fecal microbiome are close (Supplementary Fig 11a and b). Therefore, the aging-related phenotype in the fecal metabolome might be related to taxonomic and structural changes in the gut microbiota. Although the fecal microbiome and fecal metabolome appear to be linked, changes in plasma and fecal metabolites were not similar (Supplementary Fig 11c).

We further conducted an estimation of the cluster of intestinal environment matching with age using change point analysis (CPA) based on the Bayesian Information Criterion (BIC)[39]. CPA of fecal microbiome profiles showed that principal coordinate 1 (PCo1) of unweighted and weighted UniFrac was divided into 3 clusters (unweighted) including 3 to 8 weeks, 12 to 36 weeks and 40 to 72 weeks and 3 clusters (weighted) including 3 to 6 weeks, 8 to 48 weeks and 52 to 72 weeks (Supplementary Fig 12a and b). The fecal metabolome profiles of PC1 were categorized into 2 clusters, including 3 to 36 weeks and 40 to 72 weeks (Supplementary Fig 12c), which contain common gaps of 36 to 40 weeks between fecal metabolome and unweighted fecal microbiome. These data suggest that 36 to 40 weeks might be 
the critical period for the gut microbial and metabolic transition into the middle age phenotype.

Interestingly, CPA of the plasma metabolome showed no PC1 gap (Supplementary Fig 12d), implying that the transition toward host aging might occur gradually and continuously, which is different from the intestinal environment. To identify which metabolites and bacteria contribute to this grouping by random forest, we set two groups, 3 to 36 weeks and 40 to 72 weeks, which were determined by a common gap of 36 to 40 of PC1 scores of the fecal metabolome and PCo1 scores of unweighted UniFrac of the fecal microbiome (Supplementary Fig 13). Since fecal metabolites with a large contribution in the random forest contained metabolites mainly produced by gut microbiota, such as spermidine and ectoine [2,37], the changes in the fecal metabolome profile are linked to the changes in fecal microbiota, which might construct this common gap. Also, Turicibacter is at the top of the contributors of random forest and highly correlated with plasma metabolites (Supplementary Fig 10), which might influence host aging. Turicibacter has already been reported as one of the bacteria increasing with aging[29]. Another report showed that the Erysipelotrichaceae family, including Turicibacter, increased in Western-type diet-induced obese mice [40-42].

\section{Middle-age gut microbiota induces an obese phenotype in a transplanted host.}

Our network analysis highlighted the features of a middle-age murine intestinal environment, such as the increase in fecal amino acids, sugars, Erysipelotrichaceae family, and the decrease in Lachnospiraceae and Ruminococcaceae families. These features are somewhat similar to the intestinal environment of an obese phenotype[19, 40,41]. To directly ask whether the middle-age gut microbiota contributes to the obese phenotype, we orally transferred fecal microbiota from young or middle-age SPF mice into 8-weekold GF mice (Fig 4a). As expected, when placed on a high-fat diet (HFD), mice gavaged with middle-age SPF fecal microbiota displayed enhanced weight gain and higher blood glucose levels after oral glucose challenge compared to those colonized with a young SPF fecal microbiota (Fig 4b-d), although these parameters were comparable on a normal diet (Supplementary Fig. 14). Alpha diversity of the gut microbiota in the ex-GF mice gavaged with a middle-age fecal microbiota was significantly decreased after 4 weeks on a HFD (Fig 4e) and the composition of the fecal microbiota was also altered (Fig 4f). Blood insulin levels and the weight of epididymal white adipose tissue (WAT) were relatively higher but not significant, in recipients of middle-age compared to young fecal microbiota (Supplementary Fig 15). Besides, we assessed the inflammatory state of the liver, WAT and small intestine, because it was recently reported that aged gut microbiota increases intestinal permeability and promotes age-associated inflammation[7]. The expression of tumor necrosis factor a mRNA in the small intestine was significantly higher in recipients of middle-age compared to young fecal microbiota, however there was no significant difference in the expression of cytokines in the liver and WAT (Supplementary Fig 16). Taken together, these data indicate that the middle-age SPF gut microbiota has the potential to induce an obese phenotype upon high caloric intake.

\section{Discussion}


In this study, we repetitively interrogated fecal microbiome and fecal and plasma metabolome profiles from weaning to middle-age in the same mice over 72 weeks, to understand the aging-associated alterations in the gut environment. The fecal microbiome changed dynamically from weaning to young periods and then changed gradually in later periods, consistent with previous reports [20,23]. From the results of CPA, the early changing point in the fecal microbiome was at $\sim 8$ weeks; in older age, the fecal microbiome in the unweighted UniFrac data and fecal metabolome have a common gap at 36 to 40 weeks. Besides, fecal microbiome profiles were highly and significantly correlated to fecal metabolome profiles. These data indicate that the transition time point of the intestinal environment toward aging is around 36 to 40 weeks. On the other hand, the CPA of the plasma metabolome had no gap, and Procrustes analysis showed that the overall profile of plasma metabolome and fecal metabolome profiles is different, suggesting that age-related changes in the intestinal environment are not correlated with that in plasma. Previous studies have reported that inflammatory cytokines in the blood are correlated with aging in mice and humans $[7,10]$. Plasma metabolome profiles are also thought to reflect the host physiological changes with aging. Thus, our correlation analysis between fecal microbes and plasma metabolites shows that Turicibacter has a strong correlation with plasma metabolites, implying that Turicibacter may have a great impact on host physiological aging.

We found that aging modestly decreased the diversity and altered the composition of the gut microbiota. Recent metagenome studies have shown that a low fecal microbiome diversity, or dysbiosis, is associated with a variety of pathological conditions including adiposity[15-17], T2D [13, 14], colonic diseases[6, 12] and allergic diseases[43]. Alterations of the microbial composition in middle-age SPF mice were characterized by an increase in the relative abundance of the S24-7 family belonging to Bacteroidetes and Erysipelotrichaceae containing the microbial genera, Allobaculum and Turicibacter. The increase in Bacteroidetes and Turicibacter associated with aging was also observed in mouse and human studies $[18,29]$, and the increase in Erysipelotrichaceae is in agreement with previous reports of Western-type diet-induced obese mice[40-42]. Conversely, Ruminococcaceae and Lachnospiraceae were significantly decreased with aging and were highly and positively correlated with concentrations of fecal SCFA such as butyrate. Ruminococcaceae and Lachnospiraceae are members of the Clostridium cluster IV and XIVa and are known as butyrate-producing bacteria[2]. Fecal metabolome analysis showed a reduction in SCFA concentrations in the middle-age period as compared to the wean-young stage (Fig. 2g). The decrease of fecal SCFAs, in particular butyrate, has also been reported in obesity and T2D studies $[14,16,44]$. SCFAs are considered to be an important energy source for host colonic epithelium and act also as signaling molecules through the $G$ protein-coupled receptors GPR41[45] and GPR43[46, 47] to regulate energy metabolism and/or triglyceride synthesis. It is, therefore, speculated that the decrease in SCFAs in the middle-age intestinal lumen might suppress energy expenditure. Besides, recent reports on gut microbiota-mucosal immune system interactions showed that Clostridiales-derived butyrate enhances the differentiation of colonic regulatory $T$ cells, which play an important role in the suppression of immunological reactions leading to amelioration of inflammatory disorders such as allergy and colitis[48-50]. Therefore, the reduction in gut luminal butyrate due to the decrease of Clostridiales microbes in the middle-age host might also contribute to triggering these immunological 
disorders. Correlation analysis among fecal microbes and fecal metabolites also revealed that fecal glutamate and glutamine were positively and highly correlated with the Cluster 01-containing Clostridiales cluster (Supplementary Fig. 9). The decline in fecal glutamate and glutamine has been reported in previous metagenome studies in mammals including mice and humans[40]'[51]. Metagenome analysis of cecal contents from mice fed a Western-type diet has shown that genes assigned to glutamate metabolic pathways were enriched[40]. In microbiome studies across mammals, glutamate biosynthesis pathways are significantly increased in herbivore microbiomes, whereas the catabolic reactions to break down glutamate and glutamine are increased in carnivores[51]. One possibility is that the reduction of fecal glutamine and glutamate might indicate a nutrition-rich status in the gut of middleage mice, resembling that of mice fed a Westernized diet.

Plasma metabolome analysis revealed that amino acids and their derivatives were altered with age in both GF and SPF middle-age mice. Increased amino acids in human blood have been reported as a signature biomarker for the development of diabetes[52]. Also, branched-chain amino acids (BCAA) derived from food proteins contribute to the development of obesity-associated insulin resistance, as reported in animal experiments where rats were fed with HFD supplemented with BCAA[53]. A high flux rate through BCAA catabolic pathways and accumulation of glutamate may increase the transamination of pyruvate to alanine, and these highly gluconeogenic amino acids may contribute to the development of glucose intolerance in obesity[53]. Our plasma metabolome data also showed that glutamate and pyruvate concentrations were significantly higher in SPF than in GF middle-aged mice (Fig. 3h), suggesting that the changes in plasma metabolome profiles of middle-aged SPF mice could be induced by gut microbiota, which could in turn promote obesity-associated insulin resistance with aging. Moreover, alterations in the gut microbial environment with aging resulted in dysbiosis, similar to that observed in obesity and T2D. Indeed, fecal microbiota transplantation from middle-age SPF mice into young GF mice resulted in enhanced weight gain and impaired glucose tolerance compared to mice colonized with microbiota from young SPF mice, suggesting that the middle-age gut microbiota has the potential to induce an obese-type phenotype. The ability of the host to degrade and absorb proteins, lipids and polysaccharides declines with age[3-5]. This results in non-digested nutrients flowing into the lower intestine[3, 4]. In fact, the concentration of soluble starch in feces was significantly increased in middleage GF mice compared to young GF mice, as were food-derived sugars such as sucrose, maltose and maltotriose in middle-age SPF mice (Fig. 2h). Therefore, the increase of these non-digested nutrients in the lower gut of middle-age mice might lead to the nutrient-rich environment, as observed in mice fed with a Western-type diet[40-42]. As the gut microbiota attempts to adapt to these environmental changes, the result might be a decrease in microbial diversity and a higher concentration of functional metabolites such as SCFAs, and this may induce the obese-type host phenotype.

In the elderly, a reduction in intestinal mucus and a-defensin secretion influences gut microbial composition and leads to easy entry of pathogens into the mucosal layers and generation of low-grade inflammation[54]. Recent reports have shown that age-associated microbial dysbiosis in mice promotes intestinal barrier dysfunction and enhances leakage of inflammatory bacterial components into the 
circulation, which may promote increased systemic inflammation[7, 55]. These aging phenotypes are similar to HFD-induced inflammation and obesity. A HFD alters gut microbial composition and increases intestinal permeability, which leads to colonic low-grade inflammation and an increase in endotoxin levels in the intestinal lumen as well as in the plasma[56,57]. Excessive amounts of endotoxin are correlated with gut, hepatic, and adipose tissue inflammation and diabetes[56, 57].

\section{Conclusion}

In this study, we have revealed how the murine gut microbiota and their metabolites are altered with aging. Our findings provide a mechanistic understanding of the changes associated with aging in microbiome and metabolome profiles, the insight that will be crucial to develop therapeutic strategies to prevent or treat aging-associated pathologies such as obesity.

\section{Materials \& Methods}

\section{Animal experiments}

Six SPF male C57BL/6J mice (three weeks old) purchased from CLEA Japan, Inc. and three to six GF male C57BL/6J mice (three weeks old) obtained from Central Institute for Experimental Animals (CIEA, Kanagawa, Japan) were separately maintained in a vinyl isolator and fed sterilized normal diet (CA-1, CLEA Japan) until 64 (GF) or 72 weeks of age (SPF). Three of 6 GF mice were excluded at 24 weeks due to bacterial contamination of a vinyl isolator. One SPF mouse at 72 weeks and one GF mouse at 52 weeks were died (Supplementary Fig. 1). Fecal and plasma samples were collected from each mouse at the time points indicated in Supplementary Fig. 1. Samples were snap-frozen with liquid nitrogen upon collection and stored at $-80^{\circ} \mathrm{C}$ until further analysis. These long-term animal monitoring experiments were performed at CIEA and were carried out in accordance with Institutional Guidelines for Experimental Animal Welfare (09043).

\section{Fecal transplantation experiments}

GF C57BL/6N male mice (three weeks old) obtained from the RIKEN Center for Integrative Medical Sciences (Yokohama, Japan) were maintained in a vinyl isolator and fed a sterilized normal diet. Twenty milligrams of stool samples from wean-young SPF mice (3 weeks old to 12 weeks old) or middle-aged SPF mice (52 weeks-old to 72 weeks-old) were resuspended in $1 \mathrm{ml}$ of phosphate-buffered saline. Recipient mice at 8 weeks-old were gavaged with $0.2 \mathrm{ml}$ of the suspension and then separately maintained in vinyl isolators and fed with a normal diet for a week. After that, recipient mice were fed a high-fat (D12492, Research Diets) or a normal diet ad libitum for 4 weeks. We monitored body weight twice every week and collected feces every week during high-fat feeding. After high fat feeding for 4 weeks, we performed oral glucose tolerance tests (OGTT) and plasma insulin measurements. Fecal transplantation experiments were performed at Yokohama City University (Kanagawa, Japan) and were carried out in accordance with their Institutional Guidelines for Experimental Animal Welfare (T-A-17-001). 


\section{Microbiome analysis}

Fecal microbial genomic DNA was extracted from about $10 \mathrm{mg}$ of fresh feces as described previously[58]. PCR amplification of the $16 \mathrm{~S}$ rRNA gene $\mathrm{V} 1$ to $\mathrm{V} 2$ region and analysis pipeline for $16 \mathrm{~S}$ data were performed using the protocol previously reported[59].

\section{DNA sequence analyses and statistics}

Sequences were analyzed with the software package Quantitative Insights into Microbial Ecology (QIIME) [60]. The analysis removed low-quality sequences that did not perfectly match the PCR primer, were shorter than $200 \mathrm{bp}$ and longer than $400 \mathrm{bp}$, contained undetermined nucleotides (N), or did not match a barcode. Sequences were binned by barcode and clustered into de novo OTUs using a $97 \%$ similarity. Taxonomic categories were assigned using the CLASSIFIER program of the Ribosomal Database Project (RDP)[61] within QIIME. The alpha-diversity and the beta-diversity calculated with the unweighted and weighted Unifrac distance were analyzed using the QIIME software package.

\section{Metabolome analysis}

Extraction for plasma metabolites was as described previously with slight modifications[62]. A volume of $20 \mu \mathrm{l}$ of plasma was added in $230 \mu \mathrm{l}$ of methanol containing internal standards ( $20 \mu \mathrm{mol} / \mathrm{l}$ each of methionine sulfone, camphor 10 -sulfonic acid and ${ }^{13} \mathrm{C}_{6}$-d-glucose) and mixed well. Then $100 \mu$ of Milli-Q water and $250 \mu \mathrm{l}$ of chloroform were added, followed by centrifugation at 4,600 $\times g$ for $5 \mathrm{~min}$ at $4^{\circ} \mathrm{C}$. The aqueous layer was transferred to a $5-k D a$ cutoff centrifugal filter tube (Millipore) to remove large molecules. The filtrate was centrifugally concentrated at $40^{\circ} \mathrm{C}$ and reconstituted with $100 \mu \mathrm{l}$ of Milli-Q water. Fecal metabolites were extracted from fresh fecal samples (about $10 \mathrm{mg}$ ) with $400 \mu \mathrm{l} 50 \% \mathrm{MeOH}$ containing internal standards and about $100 \mathrm{mg}$ of $0.1 \mathrm{~mm}$ and two $3 \mathrm{~mm}$ zirconia/silica beads (BioSpec Products) with vigorous shaking for 3 min on a Micro Smash (TOMY). After centrifugation at 4,600 $\times g$ for $15 \mathrm{~min}$ at room temperature, the supernatant was transferred to a 5-kDa cutoff centrifugal filter tube. The filtrate was centrifugally concentrated at $40^{\circ} \mathrm{C}$ and reconstituted with $40 \mu \mathrm{l}$ of Milli-Q water. The extracted plasma and fecal samples were split into equal parts for analysis on the CE-TOFMS and LCMS/MS platforms. Ionic metabolites were analyzed using CE-TOFMS in both positive and negative mode[62]. All CE-TOFMS experiments were performed using an Agilent CE capillary electrophoresis system (Agilent Technologies, Inc.). To identify peak annotation and quantification, the obtained data were processed using in-house software (MasterHands)[63]. Concentrations of sugars in murine plasma and fecal samples were determined by LC-MS/MS in negative multiple reaction monitoring (MRM) mode on an API3000 triple quadrupole mass spectrometer (AB Sciex) as described previously[64]. All LCMS/MS data were acquired using Analyst Software (AB Sciex).

\section{Starch assay}

The concentrations of soluble starch in feces were measured by using an EnzyChrom ${ }^{\text {TM }}$ Starch Assay Kit (Funakoshi) on an Infinite M200 microplate reader (Tecan). The assays were performed following the 
manufacturer's instructions.

\section{Glucose tolerance test and plasma insulin measurement}

After $6 \mathrm{hr}$ fasting, mice were orally administrated with glucose ( $1.5 \mathrm{~g} / \mathrm{kg} \mathrm{b.w.).} \mathrm{Blood} \mathrm{glucose} \mathrm{was}$ monitored for 120 min using a glucometer (GLUCOCARD G Black, Arkray). Plasma insulin concentrations were determined using an ELISA kit (Morinaga) according to the manufacturer's instructions.

\section{Bioinformatics and statistics}

All statistical analyses were performed with GraphPad Prism version 6.0 (GraphPad Software). $P$-values $<0.05$ were considered statistically significant. PCA on fecal and plasma metabolome datasets was conducted by SIMCA-P 13.0.3. (Umetrics). Hierarchical clustering and illustrated heat map were carried out using MeV TM4 software 4.7.4. Spearman's rank correlation coefficients ( $r$ ) for all fecal microbeplasma metabolite-fecal metabolite pairs in the data across the total 118 samples were calculated by JMP version 11 (SAS Institute). Change point analysis (CPA) was performed by implementing a dynamic programming algorithm to identify optimal partitions with varying numbers of segments[65] on Mathematica 10.2. We set the minimum segment length as 2 and the maximum number of breaks as 10 . For each possible number of breaks smaller than 10 , the optimal breakpoint locations were obtained by minimizing the within-segment sums of squares. The partition achieving the lowest Bayesian information criterion (BIC) was selected. All metabolites were categorized based on the metabolic pathways of the Kyoto Encyclopedia of Genes and Genomes (KEGG) database (Supplementary Data 2).

\section{Abbreviations}

SPF: specific pathogen-free; GF: germ-free; T2D: type 2 diabetes; OTUs: operational taxonomic units; PCOA: principal coordinate analysis; HCA; hierarchical clustering analysis; CE-TOFMS: capillary electrophoresis-time-of-flight mass spectrometry; LC-MS/MS: liquid chromatography-tandem mass spectrometry; PCA: principal component; SCFAs: short-chain fatty acids; CPA: change point analysis; BIC: Bayesian Information Criterion; HFD: high-fat diet; OGTT: oral glucose tolerance tests; AUC: area under the curve; WAT: white adipose tissue; GPR: G protein-coupled receptors; BCAA: branched-chain amino acids;

\section{Declarations}

\section{Acknowledgments}

We would like to thank Drs. Peter D. Burrows and Wanping Aw for their critical reading and English editing of the manuscript. We also would like to thank Hirokuni Miyamoto, Hiroaki Kodama, Akira Watanabe, Yasunori Muraosa and Katsuhiko Kamei for their technical support of animal experiments.

\section{Authors' contributions}


Y.N., M.I., M.T. and S.F. designed the project. Y.N. performed a large part of the experiments, analyzed the data and wrote the paper. S.F. and H.O. supervised the project and wrote the paper. R.N., M.U., K.H., Y.K. T.O, A.I. and N.T. performed animal experiments. C.I., S.M. T.K. and K.S. analyzed the microbiome and metabolome data. A.H. M.S. and T.S. contributed to experimental design and data analysis. All members reviewed and edited the manuscript.

\section{Funding}

This work was supported in part by JSPS KAKENHI (17H05654 and 18H04805 to S.F., 20 K06820 and $20 \mathrm{H03010}$ to K.S. and 19K05923 to Y.N.), JST PRESTO (JPMJPR1537 to S.F.), AMED-CREST (JP19gm1010009 to S.F.), JST ERATO (JPMJER1902 to S.F.), the Takeda Science Foundation (to S.F.), the Food Science Institute Foundation (to S.F.), the Program for the Advancement of Research in Core Projects under Keio University's Longevity Initiative (to S.F.) and the Yamagata Prefectural Government and the City of Tsuruoka (to M.T.).

\section{Availability of data and materials}

16S rRNA gene sequences of gut microbiota have been deposited in the DDBJ database (http://getentry.ddbj.nig.ac.jp/) under accession number DRA010080 and DRA010096.

\section{Ethics approval and consent to participate}

Not applicable.

\section{Consent for publication}

Not applicable.

\section{Competing interests}

S.F. serves as CEO and holds equity in Metabologenomics, Inc., a company involved in the microbiomebased healthcare and drug discovery.

\section{References}

1. Lombard DB, Chua KF, Mostoslavsky R, Franco S, Gostissa M, Alt FW: DNA repair, genome stability, and aging. Cel/ 2005, 120(4):497-512. https://doi.org/10.1016/j.cell.2005.01.028.

2. Nicholson JK, Holmes E, Kinross J, Burcelin R, Gibson G, Jia W, Pettersson S: Host-gut microbiota metabolic interactions. Science (New York, NY) 2012, 336(6086):1262-1267. https://doi.org/10.1126/science.1223813.

3. Tiihonen K, Ouwehand AC, Rautonen N: Human intestinal microbiota and healthy ageing. Ageing research reviews 2010, 9(2):107-116. https://doi.org/10.1016/j.arr.2009.10.004. 
4. O'Toole PW, Claesson MJ: Gut microbiota: Changes throughout the lifespan from infancy to elderly. International Dairy Journal 2010, 20(4):281-291. https://doi.org/10.1016/j.idairyj.2009.11.010.

5. Britton E, McLaughlin JT: Ageing and the gut. The Proceedings of the Nutrition Society 2013, 72(1):173-177. https://doi.org/10.1017/S0029665112002807.

6. Swidsinski A, Ladhoff A, Pernthaler A, Swidsinski S, Loening-Baucke V, Ortner M, Weber J, Hoffmann U, Schreiber S, Dietel M et al: Mucosal flora in inflammatory bowel disease. Gastroenterology 2002, 122(1):44-54.

7. Thevaranjan N, Puchta A, Schulz C, Naidoo A, Szamosi JC, Verschoor CP, Loukov D, Schenck LP, Jury $\mathrm{J}$, Foley KP et al: Age-Associated Microbial Dysbiosis Promotes Intestinal Permeability, Systemic Inflammation, and Macrophage Dysfunction. Cell host \& microbe 2017, 21(4):455-466.e454. https://doi.org/10.1016/j.chom.2017.03.002.

8. Vaiserman AM, Koliada AK, Marotta F: Gut microbiota: A player in aging and a target for anti-aging intervention. Ageing research reviews 2017, 35:36-45. https://doi.org/10.1016/j.arr.2017.01.001.

9. Ticinesi A, Lauretani F, Milani C, Nouvenne A, Tana C, Del Rio D, Maggio M, Ventura M, Meschi T: Aging Gut Microbiota at the Cross-Road between Nutrition, Physical Frailty, and Sarcopenia: Is There a Gut-Muscle Axis? Nutrients 2017, 9(12). https://doi.org/10.3390/nu9121303.

10. Claesson MJ, Jeffery IB, Conde S, Power SE, O'Connor EM, Cusack S, Harris HM, Coakley M, Lakshminarayanan B, O'Sullivan $\mathrm{O}$ et al: Gut microbiota composition correlates with diet and health in the elderly. Nature 2012, 488(7410):178-184. https://doi.org/10.1038/nature11319.

11. O'Toole PW, Jeffery IB: Gut microbiota and aging. Science (New York, NY) 2015, 350(6265):12141215. https://doi.org/10.1126/science.aac8469.

12. Kassinen A, Krogius-Kurikka L, Makivuokko H, Rinttila T, Paulin L, Corander J, Malinen E, Apajalahti J, Palva A: The fecal microbiota of irritable bowel syndrome patients differs significantly from that of healthy subjects. Gastroenterology 2007, 133(1):24-33.

https://doi.org/10.1053/j.gastro.2007.04.005.

13. Karlsson FH, Tremaroli V, Nookaew I, Bergstrom G, Behre CJ, Fagerberg B, Nielsen J, Backhed F: Gut metagenome in European women with normal, impaired and diabetic glucose control. Nature 2013, 498(7452):99-103. https://doi.org/10.1038/nature12198.

14. Qin J, Li Y, Cai Z, Li S, Zhu J, Zhang F, Liang S, Zhang W, Guan Y, Shen D et al: A metagenome-wide association study of gut microbiota in type 2 diabetes. Nature 2012, 490(7418):55-60. https://doi.org/10.1038/nature11450.

15. Turnbaugh PJ, Ley RE, Mahowald MA, Magrini V, Mardis ER, Gordon JI: An obesity-associated gut microbiome with increased capacity for energy harvest. Nature 2006, 444(7122):1027-1031. https://doi.org/10.1038/nature05414.

16. Ridaura VK, Faith JJ, Rey FE, Cheng J, Duncan AE, Kau AL, Griffin NW, Lombard V, Henrissat B, Bain JR et al: Gut microbiota from twins discordant for obesity modulate metabolism in mice. Science (New York, NY) 2013, 341(6150):1241214. https://doi.org/10.1126/science.1241214. 
17. Turnbaugh PJ, Hamady M, Yatsunenko T, Cantarel BL, Duncan A, Ley RE, Sogin ML, Jones WJ, Roe BA, Affourtit JP et al: A core gut microbiome in obese and lean twins. Nature 2009, 457(7228):480484. https://doi.org/10.1038/nature07540.

18. Odamaki T, Kato K, Sugahara H, Hashikura N, Takahashi S, Xiao JZ, Abe F, Osawa R: Age-related changes in gut microbiota composition from newborn to centenarian: a cross-sectional study. $B M C$ microbiology 2016, 16(1):90. https://doi.org/10.1186/s12866-016-0708-5.

19. Yatsunenko T, Rey FE, Manary MJ, Trehan I, Dominguez-Bello MG, Contreras M, Magris M, Hidalgo G, Baldassano RN, Anokhin AP et al: Human gut microbiome viewed across age and geography. Nature 2012, 486(7402):222-227. https://doi.org/10.1038/nature11053.

20. Turnbaugh PJ, Ridaura VK, Faith JJ, Rey FE, Knight R, Gordon JI: The effect of diet on the human gut microbiome: a metagenomic analysis in humanized gnotobiotic mice. Science translational medicine 2009, 1(6):6ra14. https://doi.org/10.1126/scitransImed.3000322.

21. Zhou B, Yang L, Li S, Huang J, Chen H, Hou L, Wang J, Green CD, Yan Z, Huang X et al: Midlife gene expressions identify modulators of aging through dietary interventions. Proceedings of the National Academy of Sciences of the United States of America 2012, 109(19):E1201-1209. https://doi.org/10.1073/pnas.1119304109.

22. Zahn JM, Poosala S, Owen AB, Ingram DK, Lustig A, Carter A, Weeraratna AT, Taub DD, Gorospe M, Mazan-Mamczarz $\mathrm{K}$ et al: AGEMAP: a gene expression database for aging in mice. PLoS genetics 2007, 3(11):e201. https://doi.org/10.1371/journal.pgen.0030201.

23. Zhang C, Li S, Yang L, Huang P, Li W, Wang S, Zhao G, Zhang M, Pang X, Yan Z et al: Structural modulation of gut microbiota in life-long calorie-restricted mice. Nat Commun 2013, 4. https://doi.org/10.1038/ncomms3163.

24. Langille MG, Meehan CJ, Koenig JE, Dhanani AS, Rose RA, Howlett SE, Beiko RG: Microbial shifts in the aging mouse gut. Microbiome 2014, 2(1):50. https://doi.org/10.1186/s40168-014-0050-9.

25. D'Antona G, Ragni M, Cardile A, Tedesco L, Dossena M, Bruttini F, Caliaro F, Corsetti G, Bottinelli R, Carruba $\mathrm{MO}$ et al: Branched-chain amino acid supplementation promotes survival and supports cardiac and skeletal muscle mitochondrial biogenesis in middle-aged mice. Cell metabolism 2010, 12(4):362-372. https://doi.org/10.1016/j.cmet.2010.08.016.

26. Houtkooper RH, Argmann C, Houten SM, Canto C, Jeninga EH, Andreux PA, Thomas C, Doenlen R, Schoonjans K, Auwerx J: The metabolic footprint of aging in mice. Scientific reports 2011, 1:134. https://doi.org/10.1038/srep00134.

27. Landi F, Calvani R, Tosato M, Martone AM, Ortolani E, Savera G, Sisto A, Marzetti E: Anorexia of Aging: Risk Factors, Consequences, and Potential Treatments. Nutrients 2016, 8(2):69. https://doi.org/10.3390/nu8020069.

28. Cani PD, Amar J, Iglesias MA, Poggi M, Knauf C, Bastelica D, Neyrinck AM, Fava F, Tuohy KM, Chabo $C$ et al: Metabolic Endotoxemia Initiates Obesity and Insulin Resistance. Diabetes 2007, 56(7):17611772. https://doi.org/10.2337/db06-1491. 
29. van der Lugt B, Rusli F, Lute C, Lamprakis A, Salazar E, Boekschoten MV, Hooiveld GJ, Müller M, Vervoort J, Kersten $S$ et al: Integrative analysis of gut microbiota composition, host colonic gene expression and intraluminal metabolites in aging C57BL/6J mice. Aging 2018, 10(5):930-950. https://doi.org/10.18632/aging.101439.

30. Ahmadi S, Wang S, Nagpal R, Wang B, Jain S, Razazan A, Mishra SP, Zhu X, Wang Z, Kavanagh K et al: A human-origin probiotic cocktail ameliorates aging-related leaky gut and inflammation via modulating the microbiota/taurine/tight junction axis. JCl Insight 2020, 5(9). https://doi.org/10.1172/jci.insight.132055.

31. Bárcena C, Valdés-Mas R, Mayoral P, Garabaya C, Durand S, Rodríguez F, Fernández-García MT, Salazar N, Nogacka AM, Garatachea N et al: Healthspan and lifespan extension by fecal microbiota transplantation into progeroid mice. Nature medicine 2019, 25(8):1234-1242. https://doi.org/10.1038/s41591-019-0504-5.

32. Savage DC: Gastrointestinal microflora in mammalian nutrition. Annual review of nutrition 1986, 6:155-178. https://doi.org/10.1146/annurev.nu.06.070186.001103.

33. Martin FP, Dumas ME, Wang Y, Legido-Quigley C, Yap IK, Tang H, Zirah S, Murphy GM, Cloarec $\mathrm{O}$, Lindon JC et al: A top-down systems biology view of microbiome-mammalian metabolic interactions in a mouse model. Molecular systems biology 2007, 3:112. https://doi.org/10.1038/msb4100153.

34. Matsumoto M, Kibe R, Ooga T, Aiba Y, Kurihara S, Sawaki E, Koga Y, Benno Y: Impact of intestinal microbiota on intestinal luminal metabolome. Scientific reports 2012, 2:233. https://doi.org/10.1038/srep00233.

35. Koropatkin NM, Cameron EA, Martens EC: How glycan metabolism shapes the human gut microbiota. Nature reviews Microbiology 2012, 10(5):323-335. https://doi.org/10.1038/nrmicro2746.

36. Tremaroli V, Backhed F: Functional interactions between the gut microbiota and host metabolism. Nature 2012, 489(7415):242-249. https://doi.org/10.1038/nature11552.

37. Abdel-Aziz H, Wadie W, Abdallah DM, Lentzen G, Khayyal MT: Novel effects of ectoine, a bacteriaderived natural tetrahydropyrimidine, in experimental colitis. Phytomedicine : international journal of phytotherapy and phytopharmacology 2013, 20(7):585-591. https://doi.org/10.1016/j.phymed.2013.01.009.

38. Knorr S, Sinn M, Galetskiy D, Williams RM, Wang C, Müller N, Mayans O, Schleheck D, Hartig JS: Widespread bacterial lysine degradation proceeding via glutarate and L-2-hydroxyglutarate. Nature Communications 2018, 9(1):5071. https://doi.org/10.1038/s41467-018-07563-6.

39. Suzuki K, Yoshida K, Nakanishi Y, Fukuda S: An equation-free method reveals the ecological interaction networks within complex microbial ecosystems. Methods in Ecology and Evolution 2017, 8(12):1774-1785. https://doi.org/doi:10.1111/2041-210X.12814.

40. Turnbaugh PJ, Backhed F, Fulton L, Gordon JI: Diet-induced obesity is linked to marked but reversible alterations in the mouse distal gut microbiome. Cell host \& microbe 2008, 3(4):213-223. https://doi.org/10.1016/j.chom.2008.02.015. 
41. Daniel H, Gholami AM, Berry D, Desmarchelier C, Hahne H, Loh G, Mondot S, Lepage P, Rothballer M, Walker A et al: High-fat diet alters gut microbiota physiology in mice. The ISME journal2014, 8(2):295-308. https://doi.org/10.1038/ismej.2013.155.

42. Goodman AL, Kallstrom G, Faith JJ, Reyes A, Moore A, Dantas G, Gordon Jl: Extensive personal human gut microbiota culture collections characterized and manipulated in gnotobiotic mice. Proceedings of the National Academy of Sciences of the United States of America 2011, 108(15):6252-6257. https://doi.org/10.1073/pnas.1102938108.

43. Le Chatelier E, Nielsen T, Qin J, Prifti E, Hildebrand F, Falony G, Almeida M, Arumugam M, Batto JM, Kennedy $S$ et al: Richness of human gut microbiome correlates with metabolic markers. Nature 2013, 500(7464):541-546. https://doi.org/10.1038/nature12506.

44. Russell WR, Gratz SW, Duncan SH, Holtrop G, Ince J, Scobbie L, Duncan G, Johnstone AM, Lobley GE, Wallace RJ et al: High-protein, reduced-carbohydrate weight-loss diets promote metabolite profiles likely to be detrimental to colonic health. The American journal of clinical nutrition 2011, 93(5):10621072. https://doi.org/10.3945/ajcn.110.002188.

45. Samuel BS, Shaito A, Motoike T, Rey FE, Backhed F, Manchester JK, Hammer RE, Williams SC, Crowley J, Yanagisawa $\mathrm{M}$ et al: Effects of the gut microbiota on host adiposity are modulated by the short-chain fatty-acid binding G protein-coupled receptor, Gpr41. Proceedings of the National Academy of Sciences of the United States of America 2008, 105(43):16767-16772. https://doi.org/10.1073/pnas.0808567105.

46. Tolhurst G, Heffron H, Lam YS, Parker HE, Habib AM, Diakogiannaki E, Cameron J, Grosse J, Reimann F, Gribble FM: Short-chain fatty acids stimulate glucagon-like peptide-1 secretion via the G-proteincoupled receptor FFAR2. Diabetes 2012, 61(2):364-371. https://doi.org/10.2337/db11-1019.

47. Kimura I, Ozawa K, Inoue D, Imamura T, Kimura K, Maeda T, Terasawa K, Kashihara D, Hirano K, Tani $\mathrm{T}$ et al: The gut microbiota suppresses insulin-mediated fat accumulation via the short-chain fatty acid receptor GPR43. Nat Commun 2013, 4:1829. https://doi.org/10.1038/ncomms2852.

48. Arpaia N, Campbell C, Fan X, Dikiy S, van der Veeken J, deRoos P, Liu H, Cross JR, Pfeffer K, Coffer PJ et al: Metabolites produced by commensal bacteria promote peripheral regulatory T-cell generation. Nature 2013, 504(7480):451-455. https://doi.org/10.1038/nature12726.

49. Atarashi K, Tanoue T, Oshima K, Suda W, Nagano Y, Nishikawa H, Fukuda S, Saito T, Narushima S, Hase $\mathrm{K}$ et al: Treg induction by a rationally selected mixture of Clostridia strains from the human microbiota. Nature 2013, 500(7461):232-236. https://doi.org/10.1038/nature12331.

50. Furusawa Y, Obata Y, Fukuda S, Endo TA, Nakato G, Takahashi D, Nakanishi Y, Uetake C, Kato K, Kato $T$ et al: Commensal microbe-derived butyrate induces the differentiation of colonic regulatory $\mathrm{T}$ cells. Nature 2013, 504(7480):446-450. https://doi.org/10.1038/nature12721.

51. Muegge BD, Kuczynski J, Knights D, Clemente JC, González A, Fontana L, Henrissat B, Knight R, Gordon JI: Diet Drives Convergence in Gut Microbiome Functions Across Mammalian Phylogeny and Within Humans. Science (New York, NY) 2011, 332(6032):970-974. https://doi.org/10.1126/science.1198719. 
52. Wang TJ, Larson MG, Vasan RS, Cheng S, Rhee EP, McCabe E, Lewis GD, Fox CS, Jacques PF, Fernandez C et al: Metabolite profiles and the risk of developing diabetes. Nature medicine 2011, 17(4):448-453. https://doi.org/10.1038/nm.2307.

53. Newgard CB, An J, Bain JR, Muehlbauer MJ, Stevens RD, Lien LF, Haqq AM, Shah SH, Arlotto M, Slentz CA et al: A branched-chain amino acid-related metabolic signature that differentiates obese and lean humans and contributes to insulin resistance. Cell metabolism 2009, 9(4):311-326. https://doi.org/10.1016/j.cmet.2009.02.002.

54. Magrone T, Jirillo E: The interaction between gut microbiota and age-related changes in immune function and inflammation. Immunity \& ageing : / \& A 2013, 10(1):31. https://doi.org/10.1186/17424933-10-31.

55. Fransen F, van Beek AA, Borghuis T, Aidy SE, Hugenholtz F, van der Gaast-de Jongh C, Savelkoul HFJ, De Jonge MI, Boekschoten MV, Smidt H et al: Aged Gut Microbiota Contributes to Systemical Inflammaging after Transfer to Germ-Free Mice. Frontiers in immunology 2017, 8:1385. https://doi.org/10.3389/fimmu.2017.01385.

56. Chen J, He X, Huang J: Diet effects in gut microbiome and obesity. Journal of food science 2014, 79(4):R442-451. https://doi.org/10.1111/1750-3841.12397.

57. Cani PD, Delzenne NM: Interplay between obesity and associated metabolic disorders: new insights into the gut microbiota. Current opinion in pharmacology 2009, 9(6):737-743. https://doi.org/10.1016/j.coph.2009.06.016.

58. Kato T, Fukuda S, Fujiwara A, Suda W, Hattori M, Kikuchi J, Ohno H: Multiple Omics Uncovers HostGut Microbial Mutualism During Prebiotic Fructooligosaccharide Supplementation. DNA research : an international journal for rapid publication of reports on genes and genomes 2014. https://doi.org/10.1093/dnares/dsu013.

59. Sato Y, Sakurai K, Tanabe H, Kato T, Nakanishi Y, Ohno H, Mori C: Maternal gut microbiota is associated with newborn anthropometrics in a sex-specific manner. Journal of developmental origins of health and disease 2019:1-8. https://doi.org/10.1017/S2040174419000138.

60. Caporaso JG, Kuczynski J, Stombaugh J, Bittinger K, Bushman FD, Costello EK, Fierer N, Pena AG, Goodrich JK, Gordon JI et al: QIIME allows analysis of high-throughput community sequencing data. Nature methods 2010, 7(5):335-336. https://doi.org/10.1038/nmeth.f.303.

61. Wang Q, Garrity GM, Tiedje JM, Cole JR: Naive Bayesian classifier for rapid assignment of rRNA sequences into the new bacterial taxonomy. Applied and environmental microbiology 2007, 73(16):5261-5267. https://doi.org/10.1128/AEM.00062-07.

62. Hirayama A, Nakashima E, Sugimoto M, Akiyama S, Sato W, Maruyama S, Matsuo S, Tomita M, Yuzawa Y, Soga T: Metabolic profiling reveals new serum biomarkers for differentiating diabetic nephropathy. Analytical and bioanalytical chemistry 2012, 404(10):3101-3109. https://doi.org/10.1007/s00216-012-6412-x.

63. Sugimoto M, Wong DT, Hirayama A, Soga T, Tomita M: Capillary electrophoresis mass spectrometrybased saliva metabolomics identified oral, breast and pancreatic cancer-specific profiles. 
Metabolomics : Official journal of the Metabolomic Society 2010, 6(1):78-95. https://doi.org/10.1007/s11306-009-0178-y.

64. Sugimoto M, Kaneko M, Onuma H, Sakaguchi Y, Mori M, Abe S, Soga T, Tomita M: Changes in the charged metabolite and sugar profiles of pasteurized and unpasteurized Japanese sake with storage. Journal of agricultural and food chemistry 2012, 60(10):2586-2593. https://doi.org/10.1021/jf2048993.

65. Bai J, Perron P: Computation and analysis of multiple structural change models. Journal of Applied Econometrics 2003, 18(1):1-22. https://doi.org/10.1002/jae.659.

\section{Figures}




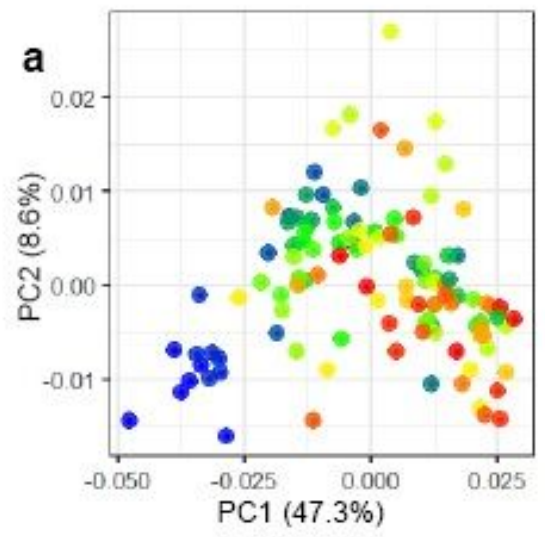

b

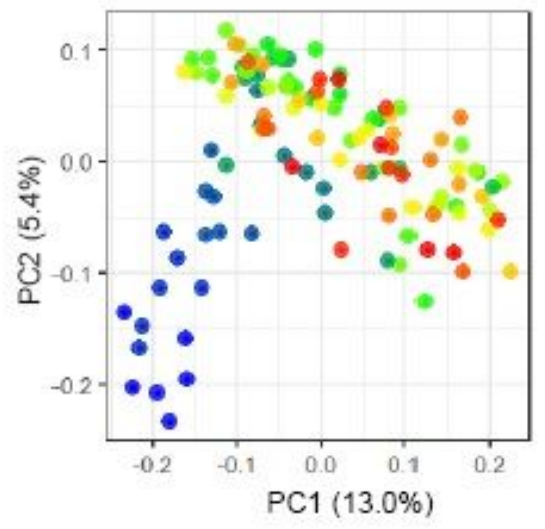

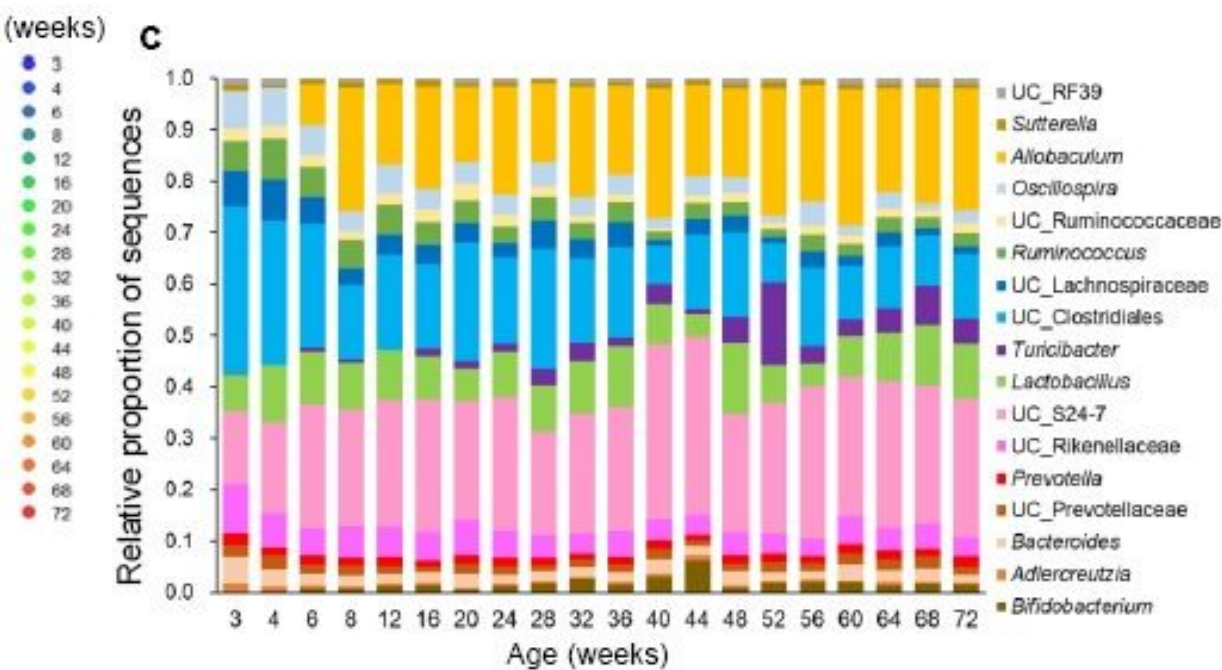

d

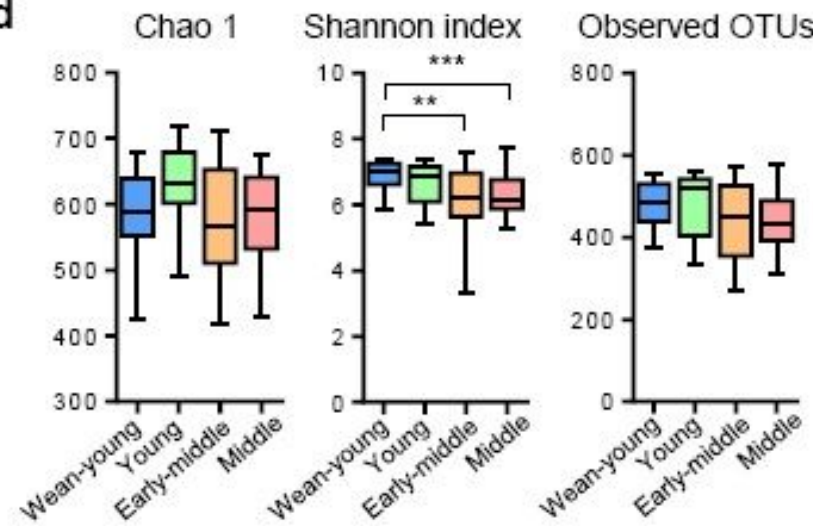

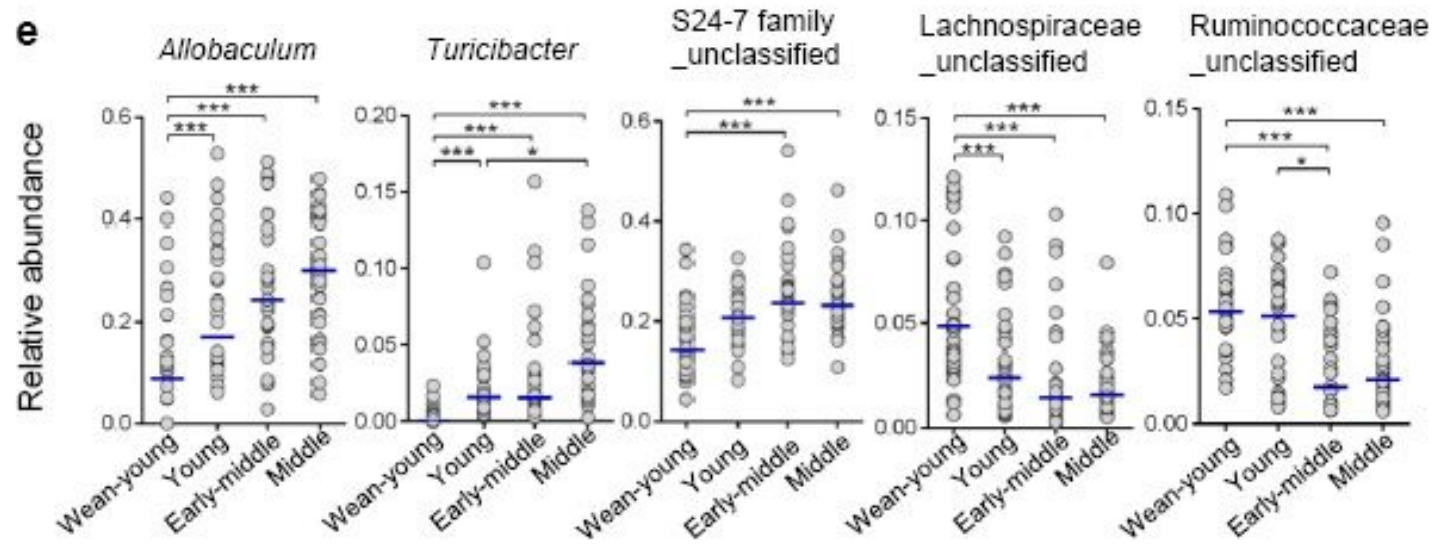

Figure 1

Age-associated alterations in the fecal microbiota PCoA of (a) weighted and (b) unweighted UniFrac distances between 16S rRNA datasets based on pyrosequencing OTUs (97\% identity) from individual fecal samples in SPF mice. Principal coordinate (PC) 1 and PC2 are the $x$-axis and y-axis, respectively. The colored dots denote the ages of mice (in weeks). (c) The distribution of genus-level taxa and relative abundance of fecal microbial communities in SPF mice. Values represent the average relative abundance 
across samples within the group at the indicated age ranges. Colors were assigned for the top 20 genera detected in total sequences with a relative abundance of $>1 \%$ in at least $75 \%$ of subjects. (d) Changes in the richness and diversity of the gut microbiota with age. Shannon diversity index numbers are shown for each group at four-time points (OTU cutoff, 97\%). The data are presented as Tukey box plots and compared at four-time points by using the Kruskal-Wallis test followed by Dunn's test: ${ }^{\star \star \star} P<0.001,{ }^{*} \mathrm{P}$ $<0.01$. (e) Dot plots of genus-level taxa with significant differences at four-time points. The dots represent each sample within the group at the indicated age ranges and the horizontal lines indicate the median values. Statistical analyses were performed using the Kruskal-Wallis test followed by Dunn's

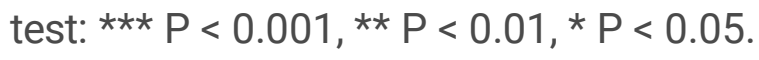




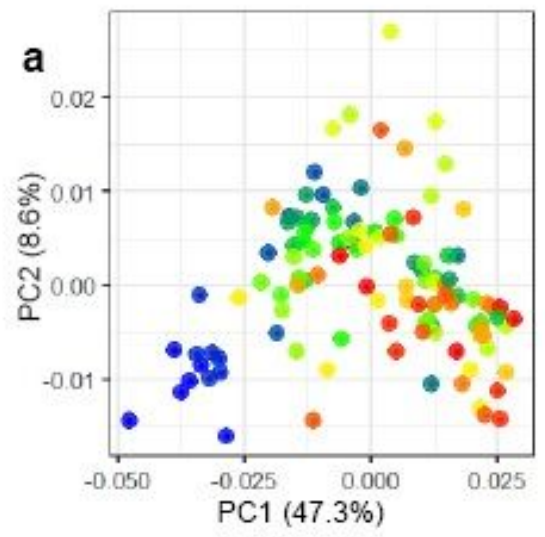

b

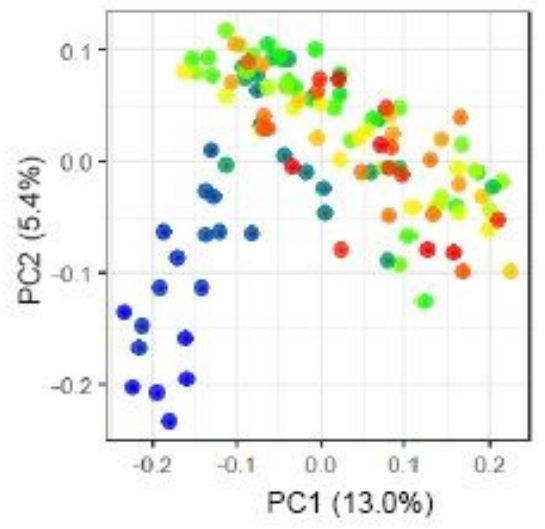

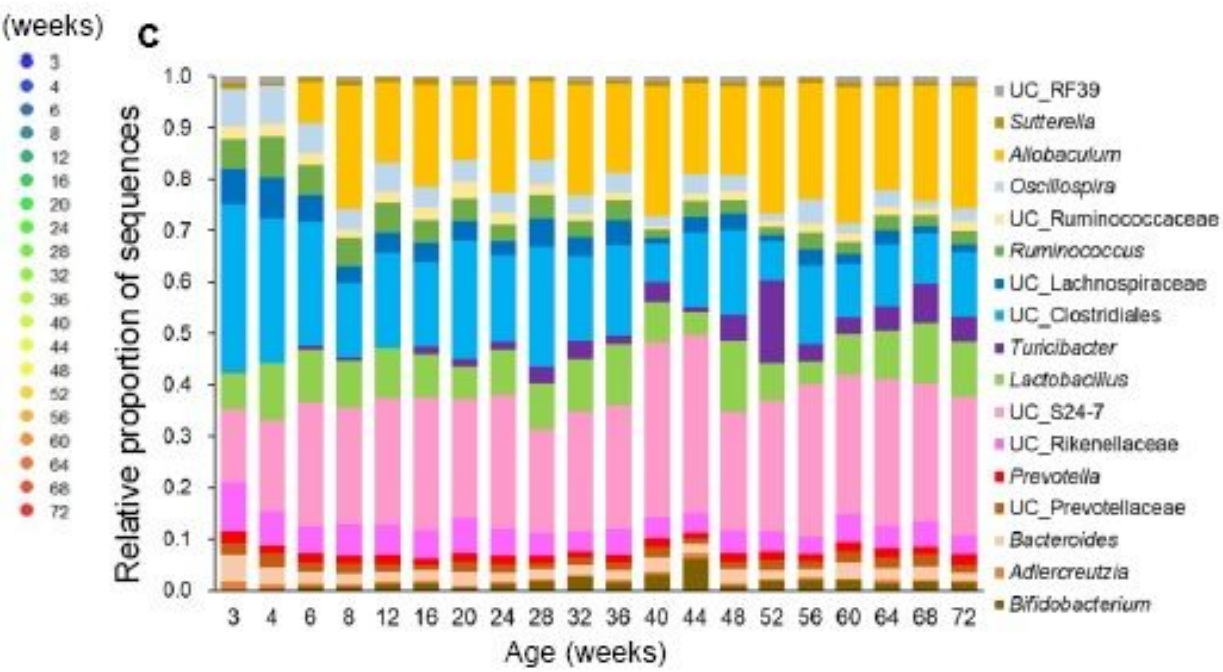

d

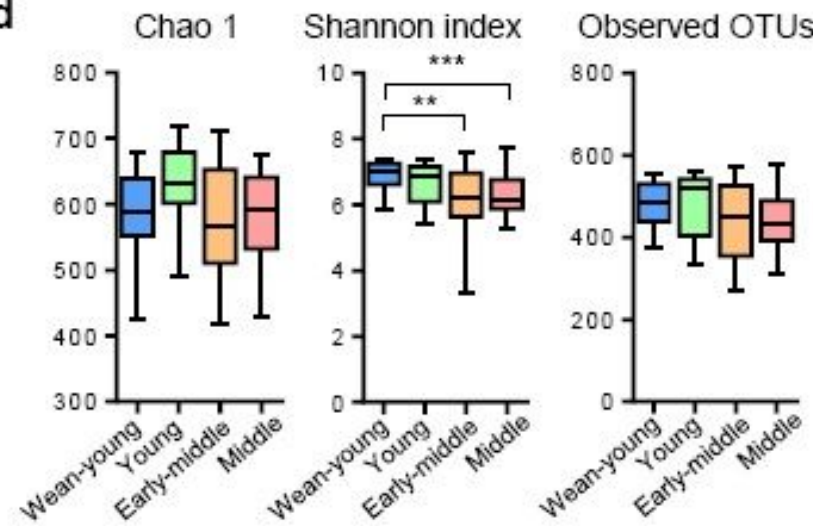

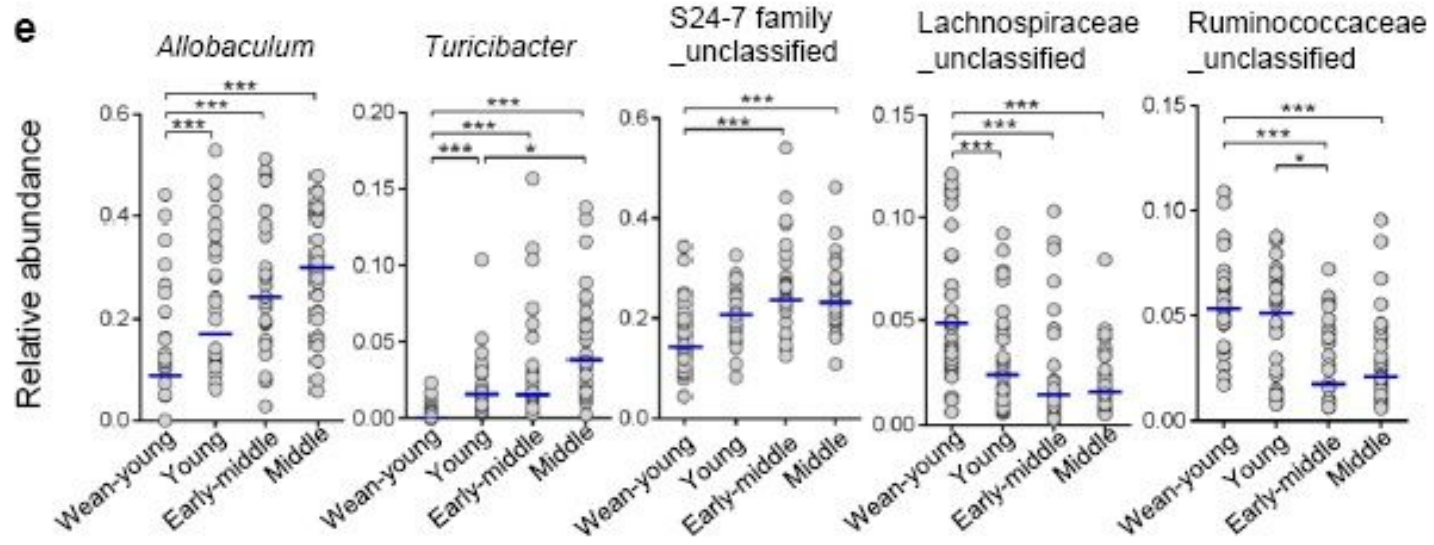

Figure 1

Age-associated alterations in the fecal microbiota PCoA of (a) weighted and (b) unweighted UniFrac distances between 16S rRNA datasets based on pyrosequencing OTUs (97\% identity) from individual fecal samples in SPF mice. Principal coordinate (PC) 1 and PC2 are the $x$-axis and y-axis, respectively. The colored dots denote the ages of mice (in weeks). (c) The distribution of genus-level taxa and relative abundance of fecal microbial communities in SPF mice. Values represent the average relative abundance 
across samples within the group at the indicated age ranges. Colors were assigned for the top 20 genera detected in total sequences with a relative abundance of $>1 \%$ in at least $75 \%$ of subjects. (d) Changes in the richness and diversity of the gut microbiota with age. Shannon diversity index numbers are shown for each group at four-time points (OTU cutoff, 97\%). The data are presented as Tukey box plots and compared at four-time points by using the Kruskal-Wallis test followed by Dunn's test: ${ }^{\star \star \star} P<0.001,{ }^{*} \mathrm{P}$ $<0.01$. (e) Dot plots of genus-level taxa with significant differences at four-time points. The dots represent each sample within the group at the indicated age ranges and the horizontal lines indicate the median values. Statistical analyses were performed using the Kruskal-Wallis test followed by Dunn's

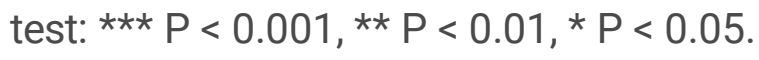



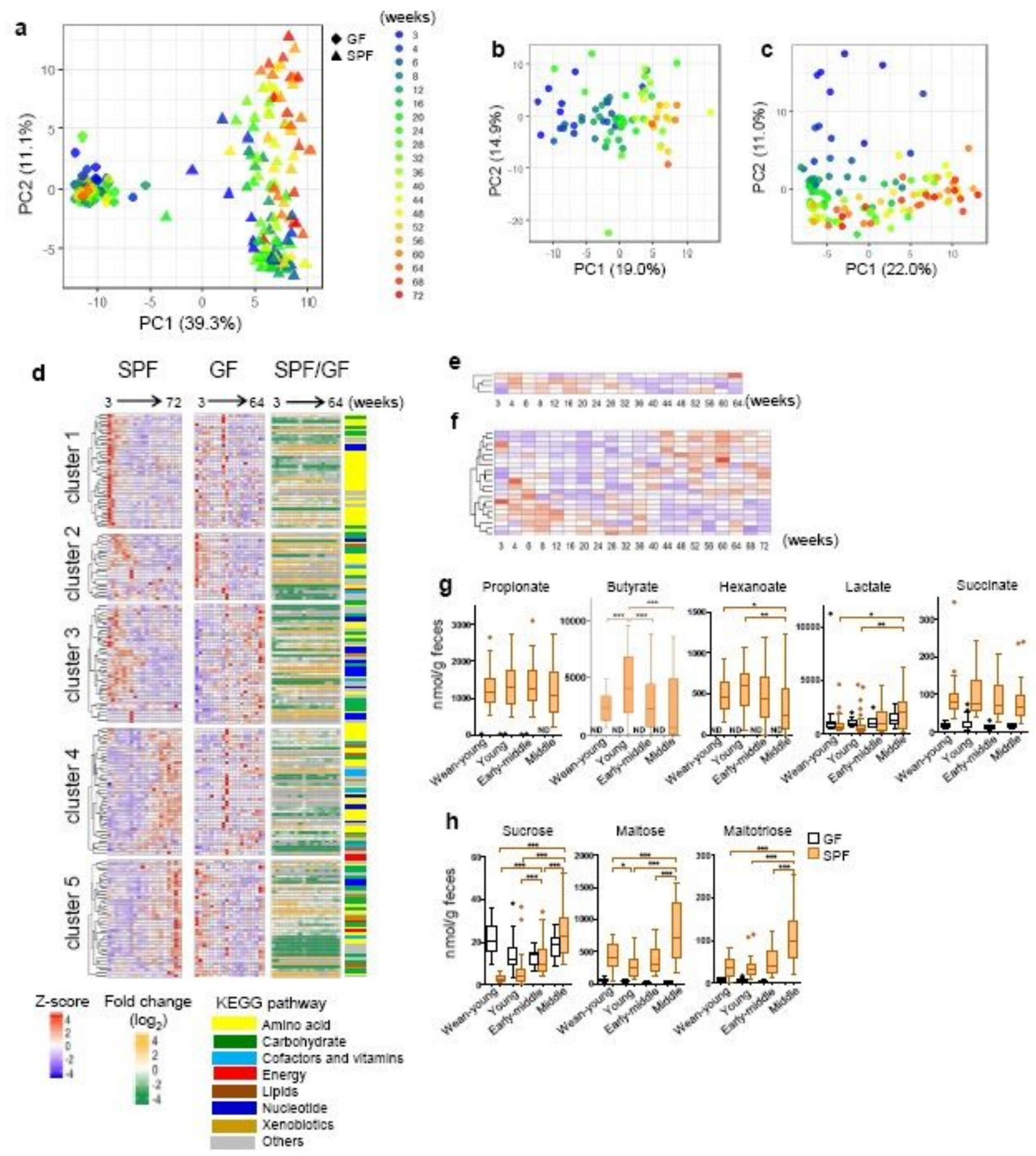

Figure 2

Fecal metabolome profiling of GF and SPF mice. PCA score plots of fecal samples from GF mice and SPF mice (a), GF mice only (b) and SPF mice only (c). Each symbol represents the principal component (PC) score from all of the individual mice. The colors indicate the age of mice in weeks, as shown on the scale located between (a) and (b). (d) Heatmaps of fecal metabolites from SPF and GF mice grouped by $\mathrm{K}$-means clustering based on a Spearman rank correlation matrix (average linkage method). Columns correspond to the samples of different ages (in weeks) from GF and SPF mice; rows correspond to 
metabolites measured by CE-TOFMS and LC-MS/MS. The colors (red to blue) indicate the average z-score values calculated for each group of mice. The heatmap on the right represents the fold-change of fecal metabolites in SPF mice compared to GF mice (orange to green). The right-most colored bars represent KEGG categories of metabolites as indicated. Heatmap of fecal metabolites from GF (e) and SPF mice (f). The colors indicate the average $z$-score values calculated for each group of mice using the same color scheme as in (d). The concentrations of fecal SCFAs and organic acids (g) and sugars (h) in GF (white boxes) and SPF (orange boxes) mice. The data are presented as Tukey box plots. Statistical differences among four age groups in SPF mice are shown (Kruskal-Wallis test followed by Dunn's test: $* \star \star ~ P<$ 0.001 , $* \star P<0.01, * P<0.05$.). No statistically significant differences were observed among GF age groups. 

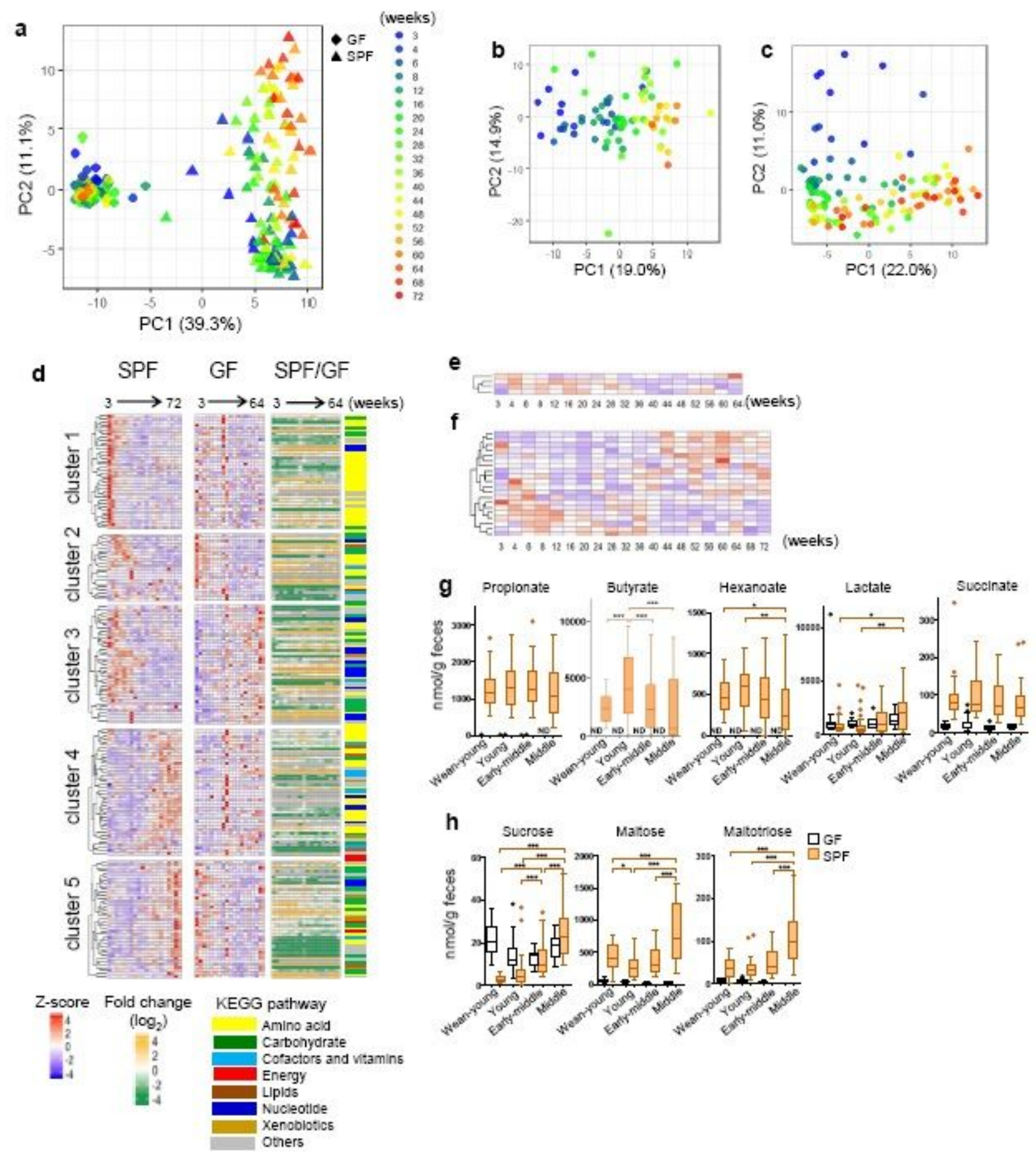

Figure 2

Fecal metabolome profiling of GF and SPF mice. PCA score plots of fecal samples from GF mice and SPF mice (a), GF mice only (b) and SPF mice only (c). Each symbol represents the principal component (PC) score from all of the individual mice. The colors indicate the age of mice in weeks, as shown on the scale located between (a) and (b). (d) Heatmaps of fecal metabolites from SPF and GF mice grouped by K-means clustering based on a Spearman rank correlation matrix (average linkage method). Columns correspond to the samples of different ages (in weeks) from GF and SPF mice; rows correspond to 
metabolites measured by CE-TOFMS and LC-MS/MS. The colors (red to blue) indicate the average z-score values calculated for each group of mice. The heatmap on the right represents the fold-change of fecal metabolites in SPF mice compared to GF mice (orange to green). The right-most colored bars represent KEGG categories of metabolites as indicated. Heatmap of fecal metabolites from GF (e) and SPF mice (f). The colors indicate the average $z$-score values calculated for each group of mice using the same color scheme as in (d). The concentrations of fecal SCFAs and organic acids (g) and sugars (h) in GF (white boxes) and SPF (orange boxes) mice. The data are presented as Tukey box plots. Statistical differences among four age groups in SPF mice are shown (Kruskal-Wallis test followed by Dunn's test: $* \star \star ~ P<$ 0.001 , $* \star P<0.01, * P<0.05$.). No statistically significant differences were observed among GF age groups. 

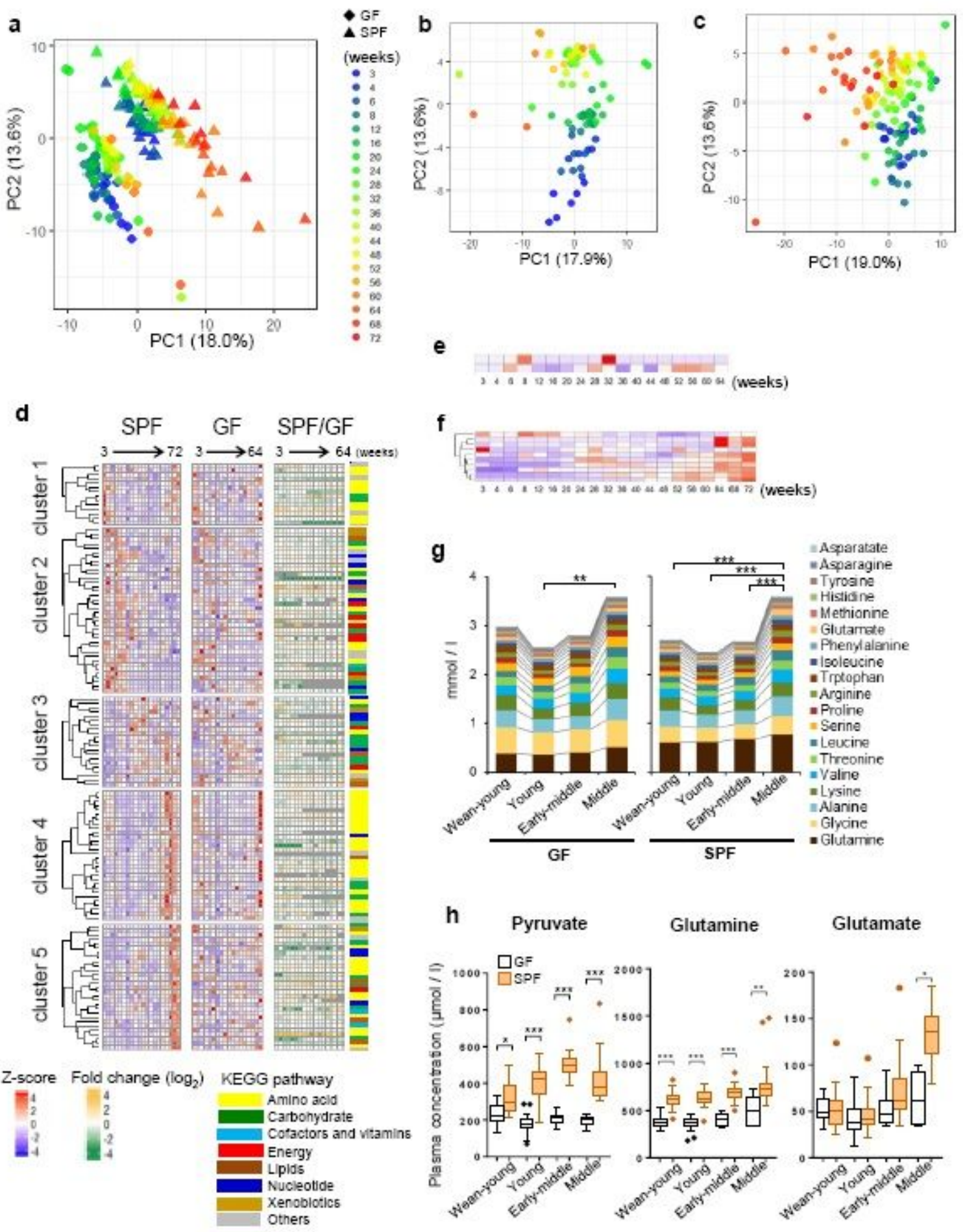

\section{Figure 3}

Plasma metabolome profiling of GF and SPF mice. PCA score plots of plasma metabolites from GF and SPF mice (a), GF mice only (b) and SPF mice only (c). Each symbol represents the mean principal component (PC) score from all of the individual mice. The colors indicate the age of mice in weeks, as shown on the scale located between (a) and (b). (d) Heatmaps of the plasma metabolites from GF and SPF mice were grouped by k-means clustering based on a Spearman rank correlation matrix (average 
linkage method). Columns correspond to the samples of different ages (in weeks) from GF and SPF mice; rows correspond to metabolites measured by CE-TOFMS and LC-MS/MS. The colors (red to blue) indicate the average $z$-score values calculated for each group of mice. The heatmap on the right represents the fold-change of plasma metabolites in SPF compared to GF mice (orange to green). The right-most colored bars represent KEGG categories of metabolites as indicated. Heatmap of the plasma metabolites from GF (e) and SPF mice (f) are shown. The colors showed the average z-score values calculated for each group of mice using the same color scheme as in (d). (g) The concentrations of total amino acids in GF and SPF mice. Statistical differences among 4 groups of mice were assessed. The data are presented

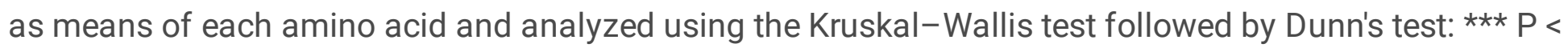
0.001 , ${ }^{*} \mathrm{P}<0.01$. (h) The concentration of plasma glutamine, pyruvate and glutamate in GF (white boxes) and SPF (orange boxes) mice described by Tukey box plots. $P$ values were calculated by MannWhitney $U$ test between GF and SPF mice: ${ }^{* *} P<0.001, * * P<0.01, * P<0.05$. 
a

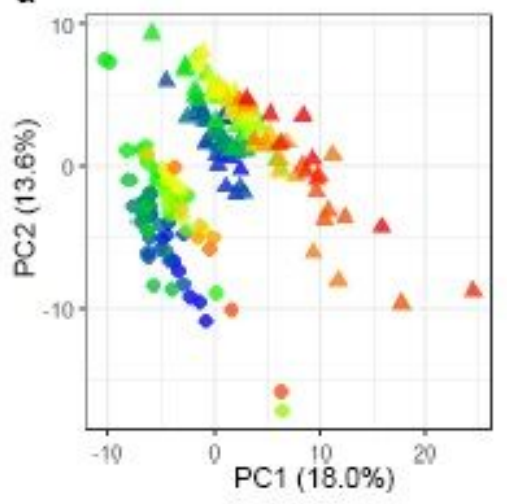

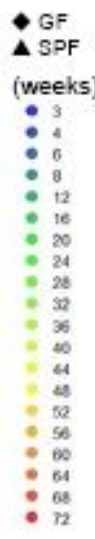

d

d

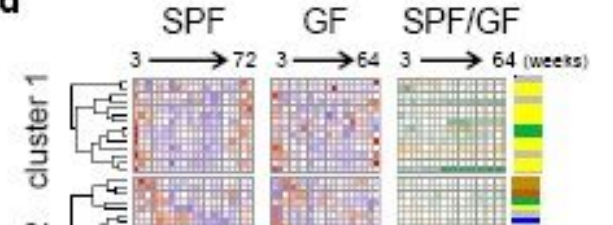

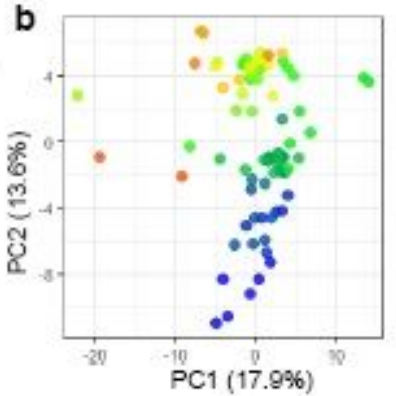

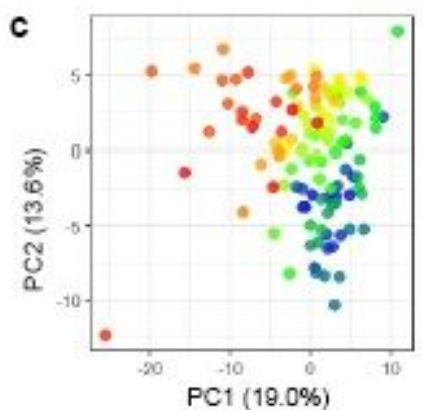

e

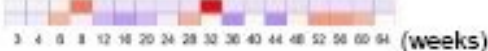

f

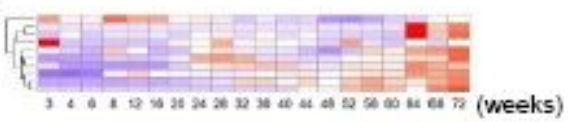

g

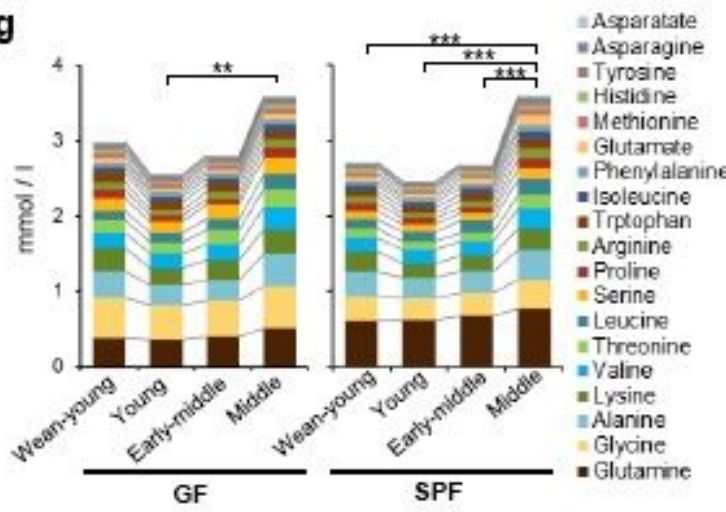

h

Pyruvate Glutamine Glutamate

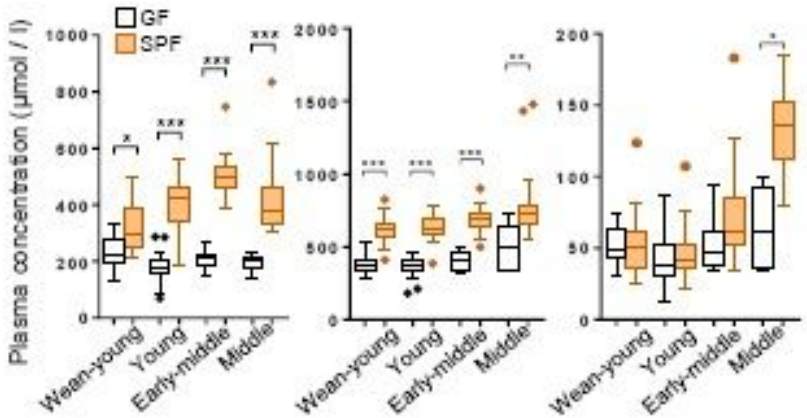

\section{Figure 3}

Plasma metabolome profiling of GF and SPF mice. PCA score plots of plasma metabolites from GF and SPF mice (a), GF mice only (b) and SPF mice only (c). Each symbol represents the mean principal component (PC) score from all of the individual mice. The colors indicate the age of mice in weeks, as shown on the scale located between (a) and (b). (d) Heatmaps of the plasma metabolites from GF and SPF mice were grouped by k-means clustering based on a Spearman rank correlation matrix (average 
linkage method). Columns correspond to the samples of different ages (in weeks) from GF and SPF mice; rows correspond to metabolites measured by CE-TOFMS and LC-MS/MS. The colors (red to blue) indicate the average $z$-score values calculated for each group of mice. The heatmap on the right represents the fold-change of plasma metabolites in SPF compared to GF mice (orange to green). The right-most colored bars represent KEGG categories of metabolites as indicated. Heatmap of the plasma metabolites from GF (e) and SPF mice (f) are shown. The colors showed the average z-score values calculated for each group of mice using the same color scheme as in (d). (g) The concentrations of total amino acids in GF and SPF mice. Statistical differences among 4 groups of mice were assessed. The data are presented

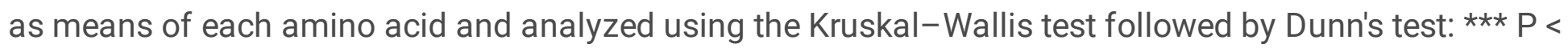
0.001 , ${ }^{*} \mathrm{P}<0.01$. (h) The concentration of plasma glutamine, pyruvate and glutamate in GF (white boxes) and SPF (orange boxes) mice described by Tukey box plots. $P$ values were calculated by MannWhitney $U$ test between GF and SPF mice: ${ }^{* *} P<0.001, * * P<0.01, * P<0.05$. 
a

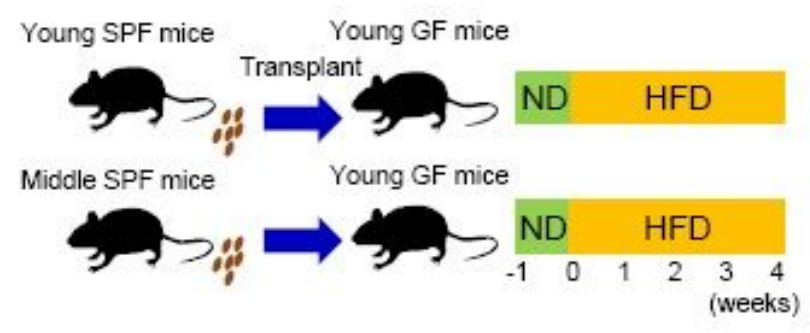

b

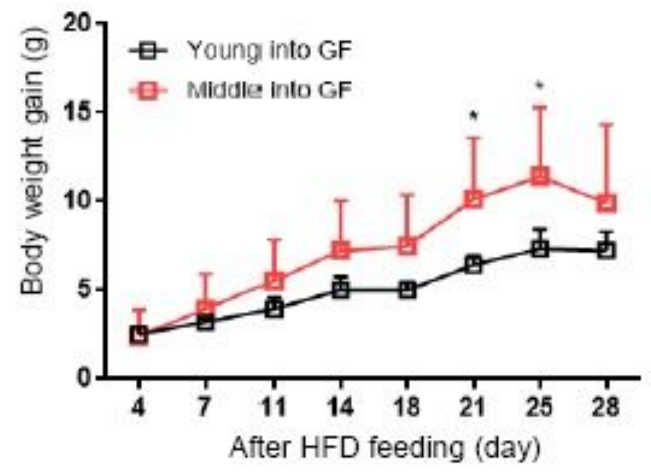

c

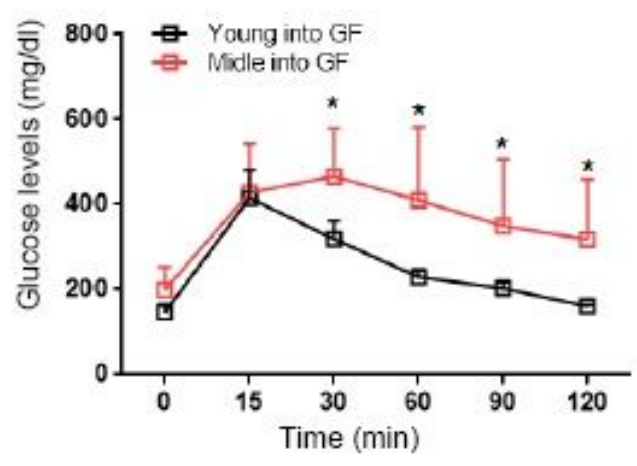

d

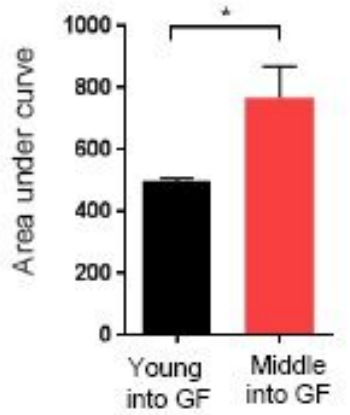

f

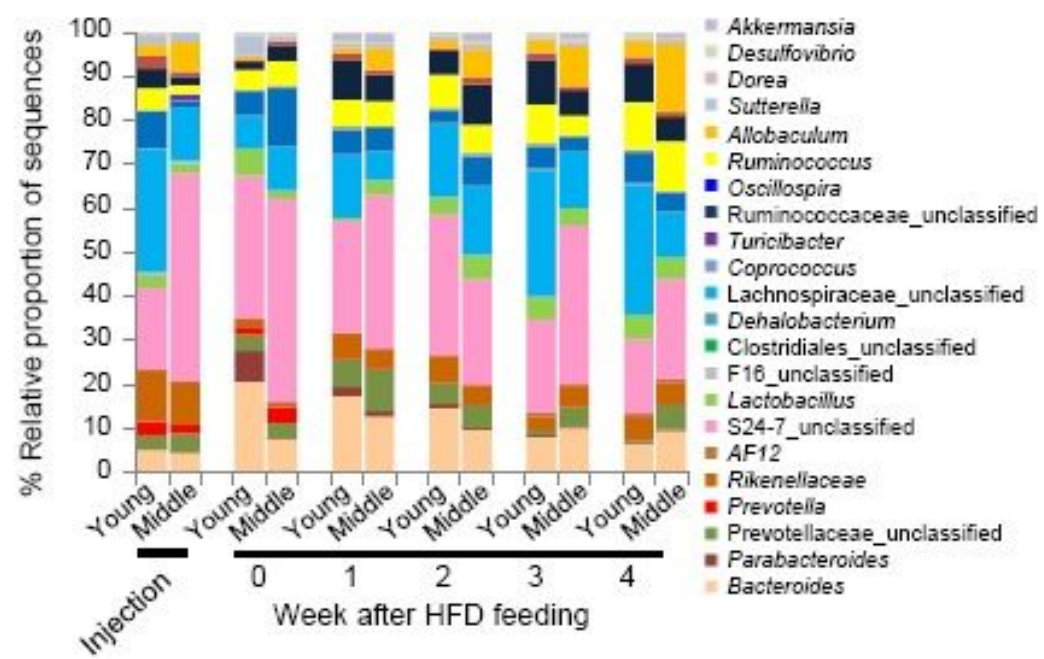

Figure 4

Middle-age gut microbiota promotes HFD induced obesity (a) Schematic overview of fecal microbiota transplantation from young or middle-age SPF mice into young GF mice. (b) Body weight gain of recipient mice transferred with fecal microbiota from young mice (black, $n=3$ ) and middle-aged mice (red, $n=6)$. (c) OGTT glucose levels and (d) area under the curve (AUC) of the OGTT at 4 weeks after HFD feeding. (e) Shannon diversity index of fecal microbiota from recipient mice along the course of HFD feeding. (f) The distribution of genus-level taxa and relative abundance of fecal microbial communities in 
recipient mice. Values represent the average relative abundance across samples within the group of recipient mice at the indicated time points. Colors were assigned for the top 20 genera (shown on the right) detected in total sequences with a relative abundance $>0.1 \%$ in at least $75 \%$ of subjects. All data are presented as mean values \pm SD and compared using the two-way ANOVA followed by Fisher's LSD: * P< 0.05 (b, c and e). The data in (d) was compared by using a t-test with Welch's correlation: ${ }^{*} \mathrm{P}<0.05$.

a

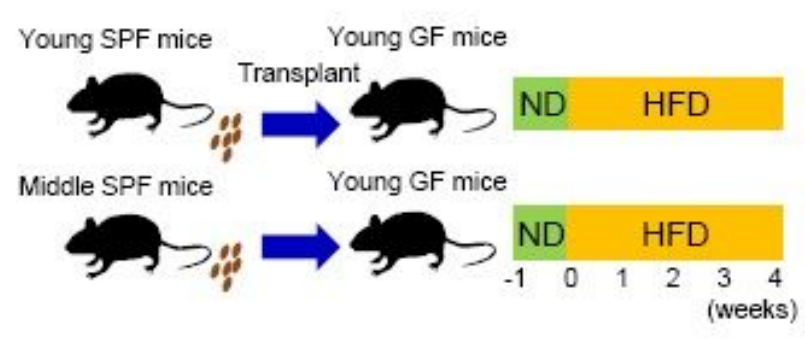

c

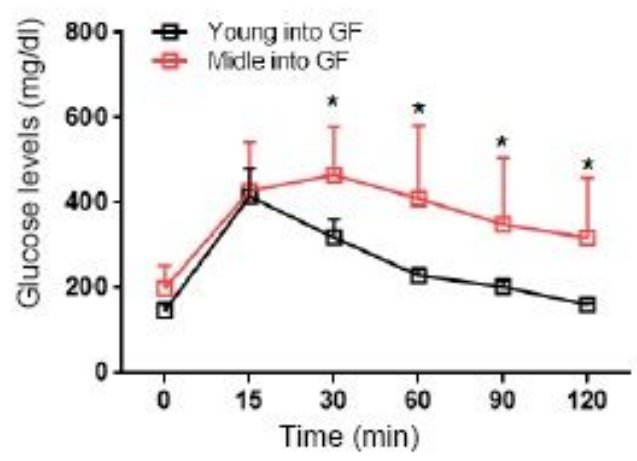

e

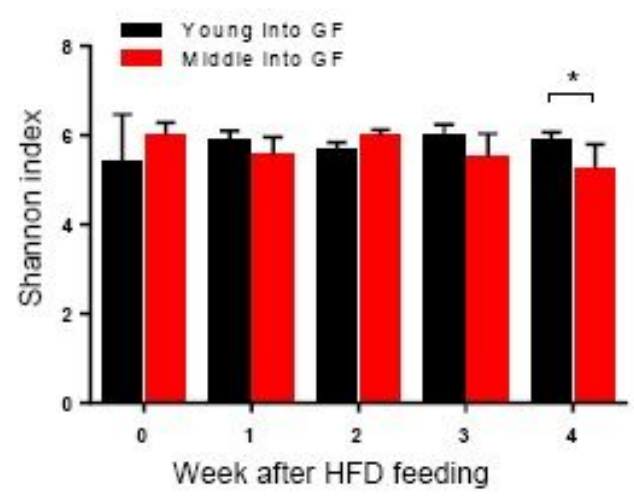

d

f b
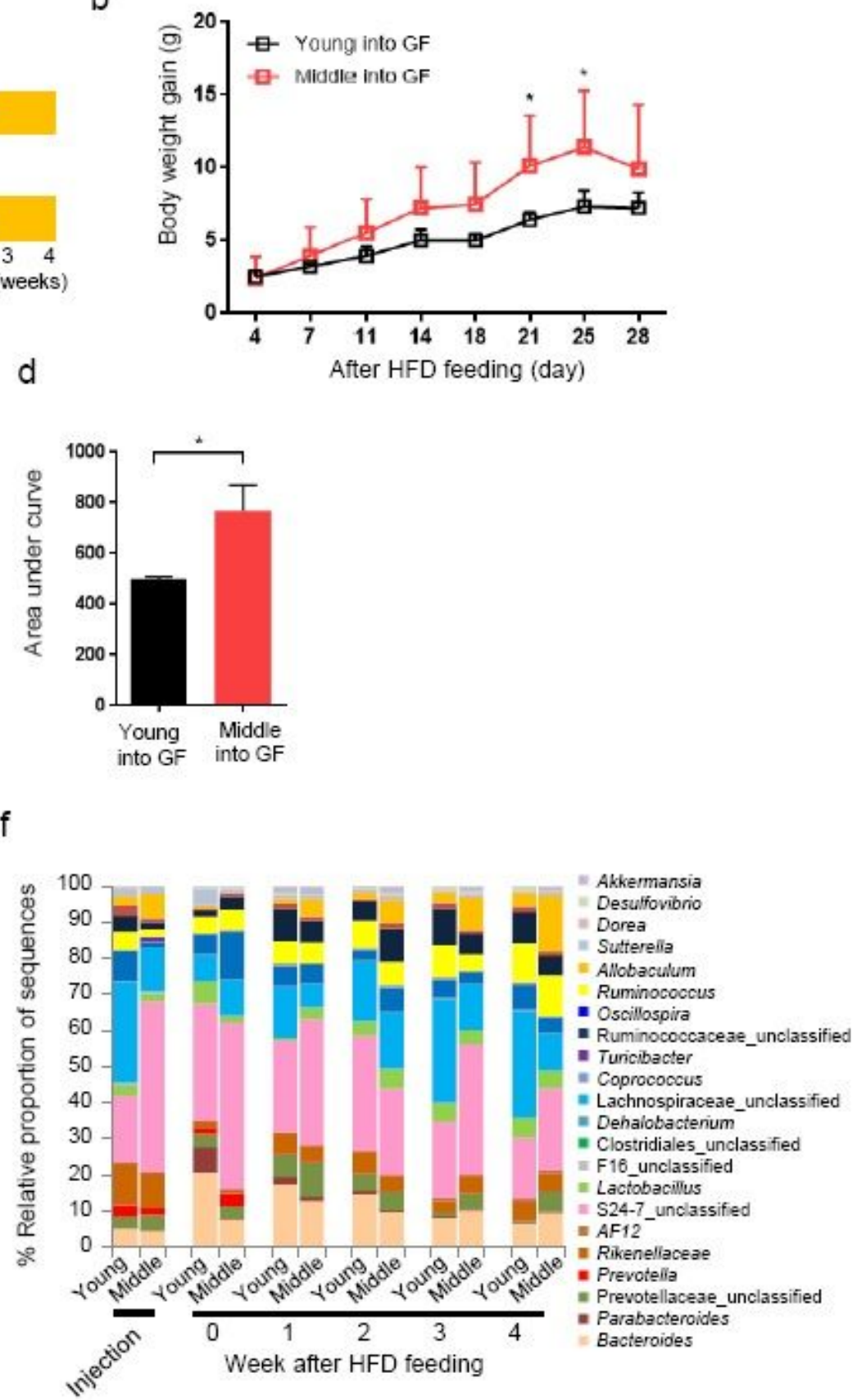

Figure 4 
Middle-age gut microbiota promotes HFD induced obesity (a) Schematic overview of fecal microbiota transplantation from young or middle-age SPF mice into young GF mice. (b) Body weight gain of recipient mice transferred with fecal microbiota from young mice (black, $n=3$ ) and middle-aged mice (red, $n=6$ ). (c) OGTT glucose levels and (d) area under the curve (AUC) of the OGTT at 4 weeks after HFD feeding. (e) Shannon diversity index of fecal microbiota from recipient mice along the course of HFD feeding. (f) The distribution of genus-level taxa and relative abundance of fecal microbial communities in recipient mice. Values represent the average relative abundance across samples within the group of recipient mice at the indicated time points. Colors were assigned for the top 20 genera (shown on the right) detected in total sequences with a relative abundance $>0.1 \%$ in at least $75 \%$ of subjects. All data are presented as mean values \pm SD and compared using the two-way ANOVA followed by Fisher's LSD: * $P<$ 0.05 (b, c and e). The data in (d) was compared by using a t-test with Welch's correlation: ${ }^{*} \mathrm{P}<0.05$.

\section{Supplementary Files}

This is a list of supplementary files associated with this preprint. Click to download.

- SupplementaryFigures.pdf

- SupplementaryFigures.pdf

- SupplementaryFig9.pdf

- SupplementaryFig9.pdf

- SupplementaryData2.xlsx

- SupplementaryData2.xlsx

- SupplementaryData1.xlsx

- SupplementaryData1.xlsx 